\title{
Importance of generalized $\mu \tau$ symmetry and its CP extension on neutrino mixing and leptogenesis
}

\author{
Rome Samanta, ${ }^{a}$ Roopam Sinha ${ }^{b}$ and Ambar Ghosal ${ }^{b}$ \\ ${ }^{a}$ Physics and Astronomy, University of Southampton, \\ University road, Southampton, SO17 1BJ, U.K. \\ ${ }^{b}$ Saha Institute of Nuclear Physics, HBNI, \\ 1/AF Bidhannagar, Kolkata 700064, India \\ E-mail: R.Samanta@soton.ac.uk, roopam.sinha@saha.ac.in, \\ ambar.ghosal@saha.ac.in
}

ABSTRACT: Within the framework of residual symmetry, two $\mathbb{Z}_{2}$ type associate $\mu \tau$ interchange symmetries robustly constrain the Dirac CP phase $\delta$ in a model independent way. Both of them predict simultaneous maximality of $\delta$ and the atmospheric mixing angle $\theta_{23}$. We show how these well known correlations will be changed if we generalize the $\mu \tau$ interchange symmetry to a $\mu \tau$ mixing symmetry. In particular, we show that the stringent condition of simultaneous maximality could be relaxed even with a very small departure from the exact $\mu \tau$ interchange. In addition, the present neutrino data on $\delta$ and $\theta_{23}$ can be explained better by the mixing symmetry. After discussing the impact of the $\mu \tau$ mixing in some realistic neutrino mass models, we show how the proposed mixing could be realized with two simultaneous CP transformations which also lead to novel and testable correlations between $\delta$ and the mixing angles $\theta_{i j}$. Next we discuss in particular, the 'three flavour regime' of leptogenesis within the CP extended framework and show, unlike the ordinary $\mathrm{CP}$ extended $\mu \tau$ interchange symmetry, a resonant leptogenesis is possible due the generalization of $\mu \tau$ interchange to the $\mu \tau$ mixing and the resulting baryon asymmetry always requires a nonmaximal $\theta_{23}$ owing to the fact that the baryon to photon ratio $\eta_{B}$ vanishes in the exact limit of $\theta_{23}=\pi / 4$. This is one of the robust predictions of this framework. The CP extended $\mu \tau$ mixing is also a novel example of a low energy effective model that provides an important insight to the off-diagonal terms of the flavour coupling matrix which have usually been neglected in literature to compute the final baryon asymmetry, in particular in the models with flavour symmetries.

Keywords: Cosmology of Theories beyond the SM, CP violation, Neutrino Physics

ARXIV EPRINT: 1805.10031 


\section{Contents}

1 Introduction 1

2 Model independent correlations in $\mu \tau$ mixing symmetry 4

2.1 Consequences of $\mathcal{G}_{1}^{g \mu \tau}$ invariance 4

$\begin{array}{ll}2.2 \text { Consequences of } \mathcal{G}_{2}^{g \mu \tau} \text { invariance } & 7\end{array}$

$2.3 \mu \tau$ mixing in some neutrino mass models 9

$\begin{array}{lll}3 & \text { CP extension of the } \mu \tau \text { mixing symmetry } & 10\end{array}$

$\begin{array}{lll}3.1 & \text { CP symmetry in a general light neutrino mass term } & 10\end{array}$

$\begin{array}{lll}3.2 & \text { CP symmetry in Type-I seesaw } & 13\end{array}$

4 Baryogenesis via leptogenesis in the CP extended framework $\quad 14$

5 Conclusions $\quad 24$

$\begin{array}{ll}\text { A General expressions for } \theta_{23} & 25\end{array}$

\section{Introduction}

In the present era of precision measurement of neutrino oscillation parameters such as the three mixing angles and two independent mass-squared differences, yet unsettled issues like the mass ordering, octant of the atmospheric mixing angle $\theta_{23}$ and the value of the Dirac CP phase $\delta$ have also drawn a lot of attention. Precise determination of the first two, for example, would confront many of the Grand Unified Theories (GUT) [1-10] of neutrino masses and mixing while a $\mathrm{CP}$ violating value of $\delta$ would have an immense implication on the observed dominance of matter over the antimatter [11-17]. Latest combined global analysis of experiments such as T2K [18, 19], NO $\nu \mathrm{A}[20-22]$, MINOS [23, 24] and RENO [25] favour a Normal Mass Ordering (NMO) at $2 \sigma[26,27]$ and shows a preference for the second octant of $\theta_{23}$ with a best-fit $\sin ^{2} \theta_{23}=0.58$. On the other hand, best fits for the phase $\delta$ are close to $284^{\circ}$ for an Inverted Mass Ordering (IMO) and $215^{\circ}$ for a NMO while the CP conserving values (i.e., $\delta=0, \pi$ ) as well as one of the $\mathrm{CP}$ violating value $\delta=\pi / 2$ are disfavoured at $70 \%$ CL and 99\% CL respectively [26]. For the NMO with solar and atmospheric masssquared differences as $\Delta m_{12}^{2}=7.39_{-0.20}^{+0.21} \times 10^{-5} \mathrm{eV}^{2}$ and $\Delta m_{31}^{2}=2.52_{-0.032}^{+0.033} \times 10^{-3} \mathrm{eV}^{2}$, global fit values of the three mixing angles and the CP phase $\delta$ are summarized in table 1.

Thus, it is a crucial juncture in regard to the measurement of $\delta$ as well as $\theta_{23}$, since many of the well-acknowledged neutrino mass models that predict, e.g., a co-bimaximal mixing $\left(\theta_{23}=\pi / 4, \delta=\pi / 2,3 \pi / 2\right)$ [28-32] would undergo a serious experimental test.

Despite having a decent theoretical understanding at least on the leading order neutrino mixing [33-35] and other testable parameters such as $\delta[36,37]$, the paradigm of residual 


\begin{tabular}{|c|cccc|}
\hline & $\theta_{12} /{ }^{\circ}$ & $\theta_{23} /{ }^{\circ}$ & $\theta_{13} /{ }^{\circ}$ & $\delta /{ }^{\circ}$ \\
\hline bf $\pm 1 \sigma$ & $33.82_{-0.76}^{+0.78}$ & $49.6_{-1.2}^{+1.0}$ & $8.61_{-0.13}^{+0.13}$ & $215_{-29}^{+40}$ \\
\hline $3 \sigma$ & $31.61 \rightarrow 36.27$ & $40.3 \rightarrow 52.4$ & $8.22 \rightarrow 8.99$ & $125 \rightarrow 392$ \\
\hline
\end{tabular}

Table 1. Best-fit, $1 \sigma$ and $3 \sigma$ ranges of three mixing angles and the Dirac CP phase $\delta$ for NMO (NuFIT [26]).

symmetry [38-43] turns out to be one of the most economical and promising approach since one really does not require the values of the elements of the light neutrino mass matrix $M_{\nu}$ to predict the mixing parameters [38, 39]. For a given neutrino Majorana mass matrix $M_{\nu}$ and a representation of the residual symmetries in the lepton flavour space $\mathcal{G}_{a}$, a horizontal invariance $\left(\mathcal{G}_{a}\right)^{T} M_{\nu} \mathcal{G}_{a}=M_{\nu}(a=1,2,3)$ together with the diagonalization condition $U^{T} M_{\nu} U=\operatorname{diag}\left(m_{1}, m_{2}, m_{3}\right)$ imply

$$
\mathcal{G}_{a}=U d_{a} U^{\dagger} \text { with }\left(d_{a}\right)_{i j}= \pm \delta_{i j}
$$

where $U$ is a unitary matrix that diagonalizes $M_{\nu}$ having nondegenerate eigenvalues. Thus the columns of the mixing matrix are simply the eigenvectors of horizontal symmetry matrix $\mathcal{G}_{a}$ with eigenvalues \pm 1 . It can be shown that out of eight possible $d_{a}$, only two are independent $[38,39]$ which lead to a closure property $\mathcal{G}_{a} \mathcal{G}_{b}=\mathcal{G}_{c}$ with $a \neq b \neq c$. Since each $\mathcal{G}$ generates a $\mathbb{Z}_{2}$ symmetry, the entire neutrino mixing could be interpreted as a consequence of a residual $\mathbb{Z}_{2} \times \mathbb{Z}_{2}$ symmetry. It also follows from eq. (1.1) that $\mathcal{G}^{2}=I, \operatorname{det} \mathcal{G}= \pm 1$. One can restrict to, without any loss of generality $\operatorname{det} \mathcal{G}=+1$ leading to two independent choices of $d$ : $d_{1}=\operatorname{diag}(1,-1,-1)$ and $d_{2}=\operatorname{diag}(-1,1,-1)$ with $d_{3}=d_{1} d_{2}$. The choice $\operatorname{det} \mathcal{G}=-1$ would then be a trivial option with all the $d$ matrices same upto an overall minus sign. Thus, given a neutrino mixing matrix $U$ and $d_{1,2}$, one can construct the corresponding $\mathbb{Z}_{2}$ generators $\mathcal{G}_{1,2}$ using eq. (1.1). For example, within the PDG convention [44], a leading order mixing matrix $U_{0}^{\mu \tau}$ could be used along with $d_{3}$ to reconstruct the well known $\mu \tau$ interchange symmetry $\mathcal{G}_{3}^{\mu \tau}[45,46]$ matrix:

$$
U_{0}^{\mu \tau}=\left(\begin{array}{ccc}
p & x & 0 \\
-\frac{x}{\sqrt{2}} & \frac{p}{\sqrt{2}} & \frac{1}{\sqrt{2}} \\
\frac{x}{\sqrt{2}} & -\frac{p}{\sqrt{2}} & \frac{1}{\sqrt{2}}
\end{array}\right) \underset{(1.1)}{\stackrel{d_{3}}{\longrightarrow}} \mathcal{G}_{3}^{\mu \tau}=\left(\begin{array}{ccc}
-1 & 0 & 0 \\
0 & 0 & 1 \\
0 & 1 & 0
\end{array}\right),
$$

where, $p \equiv \cos \theta_{12}^{\circ}$ and $x \equiv \sin \theta_{12}^{\circ}$ with $\theta_{12}^{\circ}$ being the solar mixing angle. Using eq. (1.1) and $d_{1,2}$, the other two matrices, i.e., $\mathcal{G}_{1,2}^{\mu \tau}$ can easily be constructed. We would like to call them the associate $\mu \tau$ symmetries. Since a nonzero value $\left(\sim 9^{\circ}\right)$ for the reactor mixing angle has been confirmed at more than $5.2 \sigma[47], \mathcal{G}_{3}^{\mu \tau}$ invariance must be broken in the neutrino mass term. However, due to the presence of $\mathcal{G}_{1}^{\mu \tau}\left(\mathcal{G}_{2}^{\mu \tau}\right)$ and the corresponding $d_{1}\left(d_{2}\right)$ matrix, one can simply 'rotate' $U_{0}^{\mu \tau}$ in 2-3 (1-3) plane to obtain a nonzero reactor mixing angle $\theta_{13}$ and hence the phase $\delta$. It is to be noted, that these rotations are very natural possibilities due to the degenerate eigenvalues of $\mathcal{G}_{1,2}^{\mu \tau}$ matrices. For any light neutrino mass matrix that enjoys such an invariance, the authors of refs. [40,41] showed that the phase $\delta$ turns out 
to be

$$
\begin{array}{ll}
\cos \delta=\frac{\left(s_{23}^{2}-c_{23}^{2}\right)\left(s_{12}^{2}-c_{12}^{2} s_{13}^{2}\right)}{4 c_{12} s_{12} c_{23} s_{23} s_{13}} & \text { for } \mathcal{G}_{1}^{\mu \tau}, \\
\cos \delta=\frac{\left(c_{23}^{2}-s_{23}^{2}\right)\left(c_{12}^{2}-s_{12}^{2} s_{13}^{2}\right)}{4 c_{12} s_{12} c_{23} s_{23} s_{13}} & \text { for } \mathcal{G}_{2}^{\mu \tau},
\end{array}
$$

where $\theta_{23}, \theta_{12}, \theta_{13}$ are the atmospheric, solar and reactor mixing angles respectively. For a given $3 \sigma$ range [26] of $\theta_{12}$ and $\theta_{13}$, the relations in eq. (1.3) and eq. (1.4) predict a simultaneous maximality $\left(\delta=\pi / 2\right.$ or $3 \pi / 2$ and $\left.\theta_{23}=\pi / 4\right)$. Keeping in mind that still there are no definite statements regarding the values of $\delta$ and $\theta_{23}$, we propose a generalization of the associate $\mu \tau$ interchange symmetries and we refer to them as associate $\mu \tau$ mixing symmetries. The mixing symmetry could relax the simultaneous maximality of $\delta$ and $\theta_{23}$, i.e., unlike the prediction of exact $\mu \tau$ interchange (cf. eq. (1.3)), in this scenario, nonmaximal value of $\delta\left(\theta_{23}\right)$ is allowed for a maximal value of $\theta_{23}(\delta)$. General $\mu \tau$ symmetry is basically a 'mixing' between $\mu$ and $\tau$ neutrino flavours unlike the conventional $\mu \tau$ 'interchange'. Similar to the $\mathcal{G}_{3}^{\mu \tau}$ generator (cf. eq. (1.2)), we can derive the same (we designate it as $\mathcal{G}_{3}^{g \mu \tau}$ ) for the $\mu \tau$ mixing, starting from the leading order mixing matrix $U_{0}^{g \mu \tau}$ as

$$
U_{0}^{g \mu \tau}=\left(\begin{array}{ccc}
p & x & 0 \\
-x q & p q & y \\
x y & -p y & q
\end{array}\right),
$$

where $y=\sin \theta_{g}$ and $q=\cos \theta_{g}$ with $\theta_{g}$ being the $\mu \tau$ mixing parameter and using eq. (1.1). Thus $\mathcal{G}_{3}^{g \mu \tau}$ could be constructed as

$$
\mathcal{G}_{3}^{g \mu \tau}=\left(\begin{array}{ccc}
-1 & 0 & 0 \\
0 & -\cos 2 \theta_{g} & \sin 2 \theta_{g} \\
0 & \sin 2 \theta_{g} & \cos 2 \theta_{g}
\end{array}\right) .
$$

Note that for $\theta_{g}=\pi / 4$, we recover the usual $\mu \tau$ interchange symmetry $\mathcal{G}_{3}^{\mu \tau}$ (cf. eq. (1.2)). For an elaborate discussion regarding the high energy flavour models that break down to low energy residual symmetries like $\mathcal{G}_{3}^{g \mu \tau}$, we refer to [48, 49]. Similar to [40, 41], in our proposal also, due to the vanishing $\theta_{13}$, we opt for the predictions of the associate $\mu \tau$ mixing symmetries $\mathcal{G}_{1,2}^{g \mu \tau}$ assuming that the $\mathcal{G}_{3}^{g \mu \tau}$ is broken. It is now trivial to anticipate, that the parameter $\theta_{g} \neq \pi / 4$ is entirely responsible for $\theta_{23}$ and $\delta$ not being simultaneously maximal. Having set up all the necessary prerequisites, we opt for a three step presentation of this paper. In the first step, we primarily obtain $\delta$ as $\delta \equiv f\left(\theta_{23}, \theta_{12}, \theta_{13}, \theta_{g}\right)$ (eq. (2.7) \& (2.12)) for both the associate $\mu \tau$ mixing symmetries in a model independent way. Then we present a very general numerical analysis. For example, for the given maximality of $\theta_{23}(\delta)$ we try to show how far $\theta_{g}$ could deviate from $\pi / 4$ for the allowed nonmaximal value of $\delta\left(\theta_{23}\right)$. We find that the deviation (measured by a parameter $\theta_{d}$ which is related to $\theta_{g}$ as $\theta_{g}=\pi / 4 \pm \theta_{d}$ ) cannot be very large, in particular for $\mathcal{G}_{2}^{g \mu \tau}$, the deviation is significantly small. To compare our results with exact $\mu \tau$ interchange, we then present a distribution of $\cos \delta$ for a 
small value of $\theta_{d}$ taking into account a Gaussian distribution for the other mixing angles. In the second step, we discuss how the parameter $\theta_{g}$ could be related to a realistic model parameter in neutrino mass models such as softly broken $D_{4}$ [49], Scaling Ansatz [50-56], four texture zeros in neutrino Dirac mass matrix within Type-I seesaw [58] etc. In the third step, we show how the associate mixing symmetries $\mathcal{G}_{1,2}^{g \mu \tau}$ could be a consequence of two simultaneous CP transformations [60-64] in the neutrino mass terms. We then derive novel correlations between $\delta$ and the mixing angles $\theta_{i j}$ in this class of models. Finally, from the perspective of leptogenesis, we show this CP extended $\mu \tau$ mixing symmetry is more interesting than the $\mathrm{CP}$ extended $\mu \tau$ interchange which has been a subject of recent interest in neutrino mass models [65-77]. In particular, we focus on the 'three flavour regime' of leptogenesis [78-80] and show, unlike the CP extended $\mu \tau$ interchange, a resonant leptogenesis [81] is possible in our scheme. and in this framework, a nonzero value of baryon asymmetry always requires nonmaximality in $\theta_{23}$. We also demonstrate that the $\mathrm{CP}$ extended $\mu \tau$ mixing symmetry is a novel and nice example that shows the importance of the off-diagonal terms of the flavour coupling matrix [82-85] which have usually been neglected in the computation of leptogenesis, particularly, in the models with flavour symmetries.

The rest of the paper is organized as follows. Section 2 and its various subsections deal with the explicit theoretical formalism to derive the model independent constraints and some pictorial representations of the sensitivity of the parameters $\delta$ and $\theta_{23}$ with the newly introduced parameter $\theta_{g}$ that generalizes $\mu \tau$ interchange to $\mu \tau$ mixing. We then compare our results with the exact $\mu \tau$ interchange symmetry and discuss the significance of the parameter $\theta_{d}$ in neutrino mass models such as Scaling Ansatz. In section 3 we demonstrate the $\mathrm{CP}$ extended $\mu \tau$ mixing and its consequences. In section 4 we present a qualitative as well as a quantitative description of leptogenesis within the framework of CP extended $\mu \tau$ mixing. Finally, we conclude our work in section 5.

\section{Model independent correlations in $\mu \tau$ mixing symmetry}

In this section, for both the associate mixing symmetries, first we derive analytical correlations among the Dirac $\mathrm{CP}$ violating phase, mixing angles and the proposed mixing parameter $\theta_{g}$. Then we try to show the compatibility of the scenario with recent neutrino oscillation data [26]. A systematic analysis is given in what follows.

\subsection{Consequences of $\mathcal{G}_{1}^{g \mu \tau}$ invariance}

As already introduced in the previous section, the matrix $d_{1}=\operatorname{diag}(1,-1,-1)$ has two degenerate entries. Therefore, given the symmetry $\mathcal{G}_{1}^{g \mu \tau}$ and the diagonalization condition in eq. (1.1), the second and third columns of the mixing matrix $U_{0}^{g \mu \tau}$ are not unique. It could be rotated in the 2-3 plane due to the aforementioned two-fold degeneracy. This is intriguing because the phenomenological requirement of having a nonvanishing reactor mixing angle finds a natural symmetry justification. With the choice of a most general 
unitary rotation matrix $U_{\theta}^{23}[40,41]$ in the $2-3$ plane

$$
U_{\theta}^{23}=\left(\begin{array}{ccc}
1 & 0 & 0 \\
0 & c_{\theta} & s_{\theta} e^{i \gamma} \\
0 & -s_{\theta} e^{-i \gamma} & c_{\theta}
\end{array}\right) P_{\phi}
$$

where $P_{\phi}=\operatorname{diag}\left(e^{i \phi_{1}}, e^{i \phi_{2}}, e^{i \phi_{3}}\right)$, a phenomenologically consistent PMNS matrix $U=$ $U_{0}^{g \mu \tau} U_{\theta}^{23}$ is obtained as

$$
U=\left(\begin{array}{ccc}
p & x c_{\theta} & x s_{\theta} e^{i \gamma} \\
-x q & \left(p q c_{\theta}-y s_{\theta} e^{-i \gamma}\right) & \left(y c_{\theta}+p q s_{\theta} e^{i \gamma}\right) \\
x y & -\left(p y c_{\theta}+q s_{\theta} e^{-i \gamma}\right) & \left(q c_{\theta}-p y s_{\theta} e^{i \gamma}\right)
\end{array}\right) P_{\phi}
$$

We now compare eq. (2.2) to the PMNS matrix which is parametrized according to the PDG convention [44] as

$$
U_{\mathrm{PMNS}}=P_{\chi}\left(\begin{array}{ccc}
c_{12} c_{13} & s_{12} c_{13} & s_{13} e^{-i \delta} \\
-s_{12} c_{23}-c_{12} s_{23} s_{13} e^{i \delta} & c_{12} c_{23}-s_{12} s_{23} s_{13} e^{i \delta} & c_{13} s_{23} \\
s_{12} s_{23}-c_{12} c_{23} s_{13} e^{i \delta} & -c_{12} s_{23}-s_{12} c_{23} s_{13} e^{i \delta} & c_{13} c_{23}
\end{array}\right) P_{M},
$$

where $P_{\chi}=\operatorname{diag}\left(e^{i \chi_{1}}, e^{i \chi_{2}}, e^{i \chi_{3}}\right)$ is an unphysical phase matrix and $P_{M}=\operatorname{diag}\left(1, e^{i \frac{\alpha}{2}}, e^{i \frac{\beta}{2}}\right)$ represents the Majorana phase matrix.

Comparing the (11), (12) and (13) element of eq. (2.2) and eq. (2.3), we find

$$
\begin{array}{rlrl}
c_{12} c_{13} & =p, \\
s_{12} c_{13} & =x c_{\theta}, & \chi_{1} & =\phi_{1}, \\
\frac{\alpha}{2}+\chi_{1} & =\phi_{2}, \\
s_{13} & =x s_{\theta}, & \chi_{1}-\delta+\frac{\beta}{2}-\gamma & =\phi_{3} .
\end{array}
$$

Equating the quantity $\left|U_{21}\right|^{2}-\left|U_{31}\right|^{2}$ of eq. (2.2) with the same of eq. (2.3) and using eqs. (2.4)-(2.6), we obtain

$$
\cos \delta=\frac{\left(s_{23}^{2}-c_{23}^{2}\right)\left(s_{12}^{2}-c_{12}^{2} s_{13}^{2}\right)+\cos 2 \theta_{g}\left(s_{13}^{2}+c_{13}^{2} s_{12}^{2}\right)}{4 c_{12} s_{12} c_{23} s_{23} s_{13}},
$$

where we have re-expressed $y, q$ in terms of $\theta_{g}$. As expected, for $\theta_{g}=\pi / 4$, eq. (2.7) reduces to eq. (1.3) which is the prediction of $\mathcal{G}_{1}^{\mu \tau}$. For numerical purpose, it is convenient to parametrize $\theta_{g}$ as $\theta_{g}=\pi / 4+\theta_{d}$. With this parametrization, it would be easier to realize the variation of the observables with respect to $\theta_{d}$ which is a measure of the deviation from the usual $\mu \tau$ interchange symmetry. Introduction of the mixing parameter $\theta_{g}$ now enables us to explore various interesting aspects of eq. (2.7). For example, if we set the atmospheric mixing angle $\theta_{23}$ to be maximal, the deviation of $\cos \delta$ from its maximal value can be tracked with $\theta_{d}$ from

$$
\cos \delta=\frac{\cos 2\left(\pi / 4+\theta_{d}\right)\left(s_{13}^{2}+c_{13}^{2} s_{12}^{2}\right)}{2 c_{12} s_{12} s_{13}} .
$$



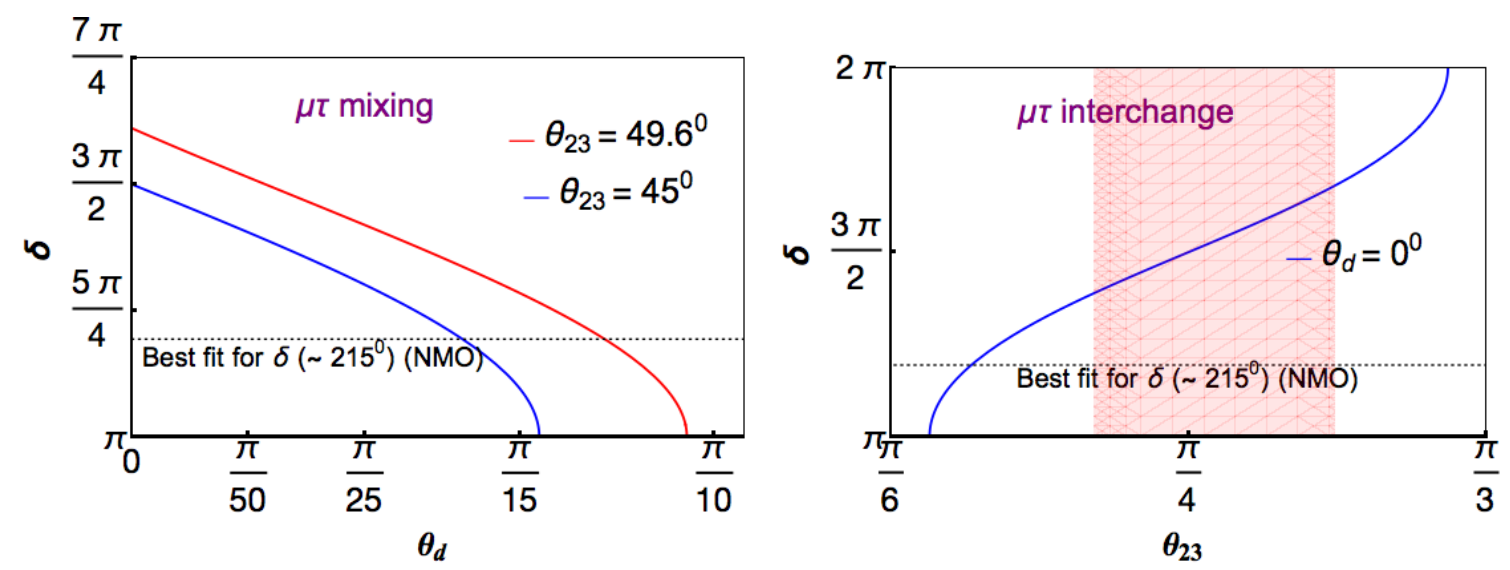

Figure 1. For $\mathcal{G}_{1}^{g \mu \tau}$ (left): variation of $\delta$ with $\theta_{d}$, where the latter is a measure of deviation from $\mu \tau$ interchange symmetry or the strength of $\mu \tau$ mixing. Here, $2 \pi-\delta$ is also an allowed solution for the same values of $\theta_{d}$. For $\mathcal{G}_{1}^{\mu \tau}$ (right): variation of $\delta$ with $\theta_{23}$. These plots are generated using the best-fit values of $\theta_{13}$ and $\theta_{12}$ for Normal Mass Ordering [26].

Similarly, for a maximal Dirac CP violation (a general expression of $\theta_{23}$ is given in the appendix), one obtains

$$
\cos 2 \theta_{23}=\frac{\cos 2\left(\pi / 4+\theta_{d}\right)\left(s_{13}^{2}+c_{13}^{2} s_{12}^{2}\right)}{s_{12}^{2}-c_{12}^{2} s_{13}^{2}} .
$$

For the best-fit values of $\theta_{12}$ and $\theta_{13}$ (table 1 ), we present a variation of $\delta$ with $\theta_{d}$ (left) for $\mu \tau$ mixing and $\delta$ with $\theta_{23}$ for $\mu \tau$ interchange ${ }^{1}$ (right) in figure 1.

It is evident from the first figure (left) in figure 1 , that the robust prediction of simultaneous maximality coming from $\mu \tau$ interchange (cf. eq. (1.3)) has now been relaxed (represented by the blue line for $\theta_{23}=45^{0}$ ) since $\theta_{d}$ can be different from zero. In fact, one can see that the deviation of $\delta$ from its maximal value is very much sensitive to $\theta_{d}$, e.g., a deviation of the former from $3 \pi / 2$ to $5 \pi / 4$ only requires a value $\approx \pi / 20$ for the latter. The red line represents the variation of $\delta$ for the current best-fit value $\theta_{23}=49.6^{0}$ for NMO. As one can see, in the $\mu \tau$ interchange limit $\left(\theta_{d}=0\right)$ best-fit of $\theta_{23}$ is not consistent with the current best-fit of $\delta=215^{0}$ (represented by the horizontal black dotted line). However, in the proposed $\mu \tau$ mixing scheme, one can fit the best-fits simultaneously just by tuning a single parameter $\theta_{d}(\sim \pi / 12)$ as shown by the red line. The figure in the right hand side shows a variation of $\delta$ with $\theta_{23}$ for the exact $\mu \tau$ interchange symmetry. It is interesting to notice, that even within the $3 \sigma$ range of $\theta_{23}$, one cannot reconcile the best-fit value of $\delta$. Thus from the viewpoint of current experimental results, the proposed $\mathcal{G}_{1}^{g \mu \tau}$ is a more admissible symmetry than the $\mathcal{G}_{1}^{\mu \tau}$. This can also be realized more clearly from the figure 2 where we present a statistical comparison between the predictions of $\mu \tau$ interchange $\left(\mathcal{G}_{1}^{\mu \tau}\right)$ and $\mu \tau$ mixing $\left(\mathcal{G}_{1}^{g \mu \tau}\right)$. The probability density $\operatorname{plot}^{2}$ in the left hand side of figure 2 shows,

\footnotetext{
${ }^{1}$ From now on when we address predictions of $\mu \tau$ mixing or $\mu \tau$ interchange, it could be assumed that we are implying the predictions for the associate symmetries.

${ }^{2}$ These are basically density-histogram plots. As one goes from the blue region to the red one, the number density of points increases, as shown in the bar-legends.
} 

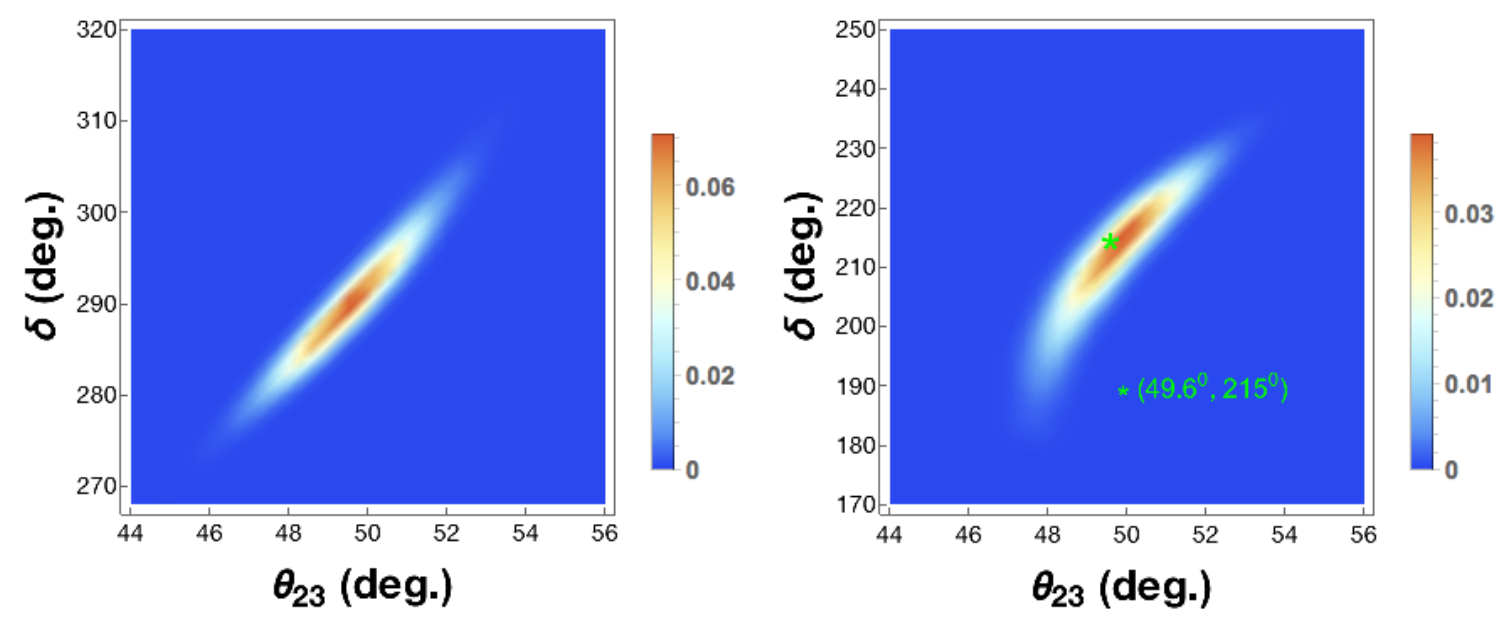

Figure 2. For $\mathcal{G}_{1}^{\mu \tau}$ (left): probability distribution of $\delta$ with $\theta_{23}$. For $\mathcal{G}_{1}^{g \mu \tau}$ (right): the same plot but for $\theta_{d}=\pi / 12$. Here we have used Gaussian distribution for each of the mixing angles.

for the $\mathcal{G}_{1}^{\mu \tau}$, most probable values of $\delta$ lie within a region centred approximately around $\delta \sim 290^{\circ}$ which is far away (tension is $\sim 2 \sigma$ ) from the best-fit $215^{0}$ for NMO. On the other hand, as also explained earlier, for the best-fit of $\theta_{23}$, the most probable values of $\delta$ could be reconciled with the best-fit $215^{0}$ for $\theta_{d}=\pi / 12$. As shown in right panel of figure 2 , the entire pattern (shown in the left side) has shifted near the best-fit shown by the green ' $*$ '. Before discussing the consequences of $\mathcal{G}_{2}^{g \mu \tau}$, let us quickly address some important points. First of all, when we say the $\mu \tau$ interchange $\left(\mathcal{G}_{1}^{\mu \tau}\right)$ is disfavoured, we always mean a NMO. As one can see from figure 2 (left hand side), best-fit of $\delta\left(=284^{0}\right)$ for IMO could be well reconciled within $1 \sigma$ of $\theta_{23}$. However, as mentioned in the introduction, that the IMO seems to be disfavoured now by the current experimental data. One might also wonder how the mixing parameter $\theta_{g}=\pi / 4 \pm \theta_{d}$ could be realized in a realistic neutrino mass model. Because so far it appears to be a model independent tuning parameter, except the mention to refs. $[48,49]$ in the introduction. In section 2.3 , we shall briefly discuss some models regarding the relation of $\theta_{g}$ with the model parameters and show indeed there is a large class of models that exhibit $\mu \tau$ mixing at low energy.

\subsection{Consequences of $\mathcal{G}_{2}^{g \mu \tau}$ invariance}

In this case, a rotation in 1-3 plane is possible due to the degeneracy in $d_{2}=(-1,1,-1)$ matrix. By choosing a most general unitary rotation matrix $U_{\theta}^{13}[40,41]$ as

$$
U_{\theta}^{13}=\left(\begin{array}{ccc}
c_{\theta} & 0 & s_{\theta} e^{i \gamma} \\
0 & 1 & 0 \\
-s_{\theta} e^{-i \gamma} & 0 & c_{\theta}
\end{array}\right) P_{\phi}
$$



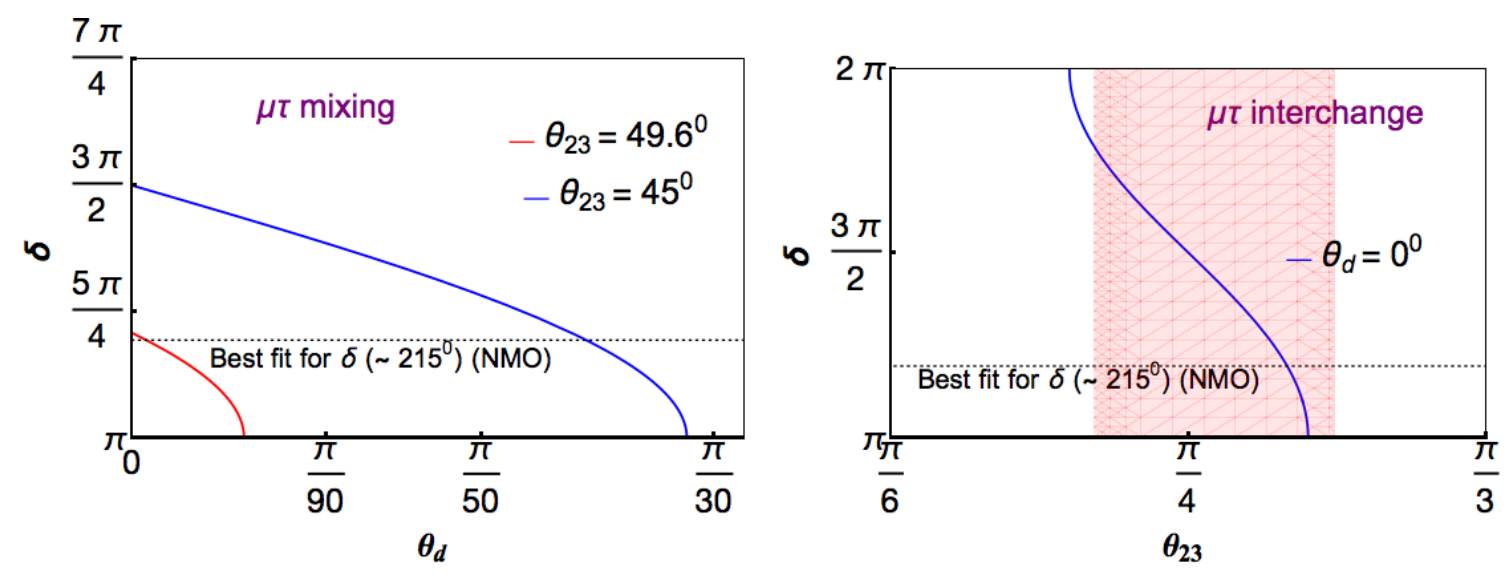

Figure 3. For $\mathcal{G}_{2}^{g \mu \tau}$ (left): variation of $\delta$ with $\theta_{d}$, where the latter is a measure of deviation from $\mu \tau$ interchange symmetry or the strength of $\mu \tau$ mixing. Here, $2 \pi-\delta$ is also an allowed solution for the same values of $\theta_{d}$. For $\mathcal{G}_{2}^{\mu \tau}$ (right): variation of $\delta$ with $\theta_{23}$. These plots are generated using the best-fit values of $\theta_{13}$ and $\theta_{12}$ for Normal mass ordering [26].

we construct the phenomenologically viable PMNS matrix $U=U_{0}^{g \mu \tau} U_{\theta}^{13}$ which is given by

$$
U=\left(\begin{array}{ccc}
p c_{\theta} & x & p s_{\theta} e^{i \gamma} \\
-\left(x q c_{\theta}+y s_{\theta} e^{-i \gamma}\right) & p q & \left(y c_{\theta}-x q s_{\theta} e^{i \gamma}\right) \\
\left(x y c_{\theta}-q s_{\theta} e^{-i \gamma}\right) & -p y & \left(q c_{\theta}+x y s_{\theta} e^{i \gamma}\right)
\end{array}\right) P_{\phi} .
$$

Following a similar procedure demonstrated previously for $\mathcal{G}_{1}^{g \mu \tau}$, we compare eq. (2.11) with the PMNS matrix of eq. (2.3) and equate the quantity $\left|U_{22}\right|^{2}-\left|U_{32}\right|^{2}$ of both the matrices. This results in

$$
\cos \delta=\frac{\left(c_{23}^{2}-s_{23}^{2}\right)\left(c_{12}^{2}-s_{12}^{2} s_{13}^{2}\right)-\cos 2 \theta_{g}\left(s_{13}^{2}+c_{13}^{2} c_{12}^{2}\right)}{4 c_{12} s_{12} c_{23} s_{23} s_{13}} .
$$

Thus for $\theta_{g}=\pi / 4$, we recover eq. (1.4). In this case, parametrizing ${ }^{3} \theta_{g}=\pi / 4-\theta_{d}$, one can derive the simple correlations of $\delta$ and $\theta_{23}$ with $\theta_{g}$ as

$$
\begin{aligned}
\cos \delta & =\frac{-\cos 2\left(\pi / 4-\theta_{d}\right)\left(s_{13}^{2}+c_{13}^{2} c_{12}^{2}\right)}{2 c_{12} s_{12} s_{13}}, \\
\cos 2 \theta_{23} & =\frac{\cos 2\left(\pi / 4-\theta_{d}\right)\left(s_{13}^{2}+c_{13}^{2} c_{12}^{2}\right)}{c_{12}^{2}-s_{12}^{2} s_{13}^{2}}
\end{aligned}
$$

for a maximal atmospheric angle and maximal Dirac CP violation respectively.

In figure 3 (left figure), the red line represents the variation of $\delta$ with $\theta_{d}$ for the best-fit of $\theta_{23}=49.6^{0}$. We find a remarkable 'coincidence' of eq. (1.4) with the present data on $\theta_{23}$ and $\delta$. It is evident from the plot in the left panel of figure 3 (also in figure 4), one needs really a tiny departure (numerically, $\theta_{d}$ less than $0.5^{0}$ ) from exact $\mu \tau$ interchange to fit the most probable values of $\delta$ simultaneously with the best-fit of $\delta$ and $\theta_{23}$. When we opt

\footnotetext{
${ }^{3}$ Unlike the case of $\mathcal{G}_{1}^{g \mu \tau}$, here we parametrize $\theta_{g}$ as $\theta_{g}=\pi / 4-\theta_{d}$ instead of $\theta_{g}=\pi / 4+\theta_{d}$, just to show the variation of $\delta$ with the positive values of $\theta_{d}$.
} 

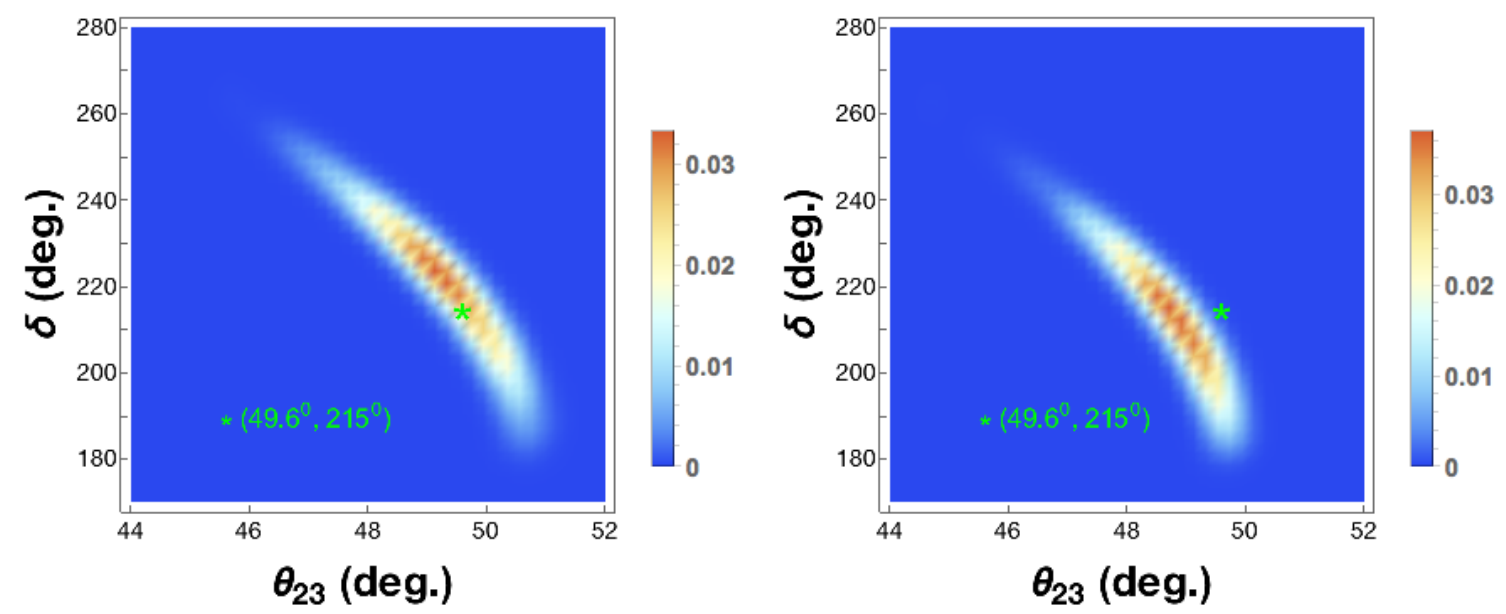

Figure 4. For $\mathcal{G}_{2}^{\mu \tau}$ (left): probability distribution of $\delta$ with $\theta_{23}$. For $\mathcal{G}_{2}^{g \mu \tau}$ (right): the same plot but for $\theta_{d}=\pi / 180$. Here we have used Gaussian distribution for each of the mixing angles.

for a larger departure from $\mu \tau$ interchange, even with $\theta_{d}=1^{0}$, the most probable values of $\delta$ start to move towards CP conserving values as shown in the right panel of figure 4 . Thus, as far as the current data on $\delta$ and $\theta_{23}$ is concerned, undoubtedly, $\mu \tau$ interchange (here $\mathcal{G}_{2}^{\mu \tau}$ ) is a better symmetry to explain the data than the proposed $\mu \tau$ mixing (here $\left.\mathcal{G}_{2}^{g \mu \tau}\right)$. However, compared to the previously released data [27], the new data [26] shows a tendency to move towards the $\mathrm{CP}$ conserving values mainly driven by $\mathrm{NO} \nu \mathrm{A}$ anti-neutrino appearance channel [26]. If this trend continues, one has to think beyond $\mu \tau$ interchange symmetry. In that case, the $\mu \tau$ mixing (as shown in the right panel of figure 4 ) could be a good option to explain the data.

So we conclude this section with the remark that, to explain the present data, the proposed $\mathcal{G}_{1}^{g \mu \tau}$ (mixing) symmetry has an edge over the $\mathcal{G}_{1}^{\mu \tau}$ (interchange), whereas the symmetries which are in the class of $\mathcal{G}_{2}$, to explain the present data, the interchange scenario is better than the proposed mixing scenario.

\section{$2.3 \mu \tau$ mixing in some neutrino mass models}

In this section, we discuss some examples of the low energy residual $\mu \tau$ symmetry so that the parameter $\theta_{g}$ could be connected to the model parameter(s). Indeed there is a large class of models that belong to the mixing category. For example the authors of refs. [48, 49] derive the mixing from explicit symmetry group $D_{4}$ where the $\mu \tau$ mixing parameter $\theta_{g}$ could be related to the model parameter as

$$
\cos 2 \theta_{g} \sim-\frac{\mu_{\text {soft }}}{M}
$$

where $\mu_{\text {soft }}$ is a soft breaking term in the $D_{4}$ model and $M$ is the mass scale of the $\mathrm{RH}$ neutrinos needed to obtain Type-I seesaw light neutrino masses. The model predicts the same leading order mixing matrix as shown in eq. (1.5). Now to generate a nonzero $\theta_{13}$ we can further add soft breaking terms to the model. However, notice that, here the introduction of new breaking terms corresponds to the rotation of the leading order mixing 
matrix (cf. eq. (2.1)) and the final prediction (cf. eq. (2.7)) is independent of the rotation angle. Since in general, generation of nonzero $\theta_{13}$ requires small breaking terms, eq. (2.15) still holds at the leading order. But unlike the $D_{4}$ model, $\cos 2 \theta_{g}$ is not directly related only to $\theta_{23}$ but it connects $\theta_{23}$ and $\delta$ with the correlations shown in eq. (2.7) or eq. (2.12). The authors of refs. [48, 49] conclude, that to test a sizeable deviation of physical parameters such as $\theta_{23}$ one needs the scale of $\mu_{\text {soft }}$ of same order as the mass scale of the $\mathrm{RH}$ neutrinos requiring a large deviation from $\mu \tau$ interchange $\left(\cos 2 \theta_{g} \simeq 1\right)$. However, as we have shown in the previous section, even with a small departure from the interchange symmetry, one can test the parameters $\delta$ and $\theta_{23}$ with the correlations derived in this paper. Thus the scale of $\mu_{\text {soft }}$ and $M$ need not be the same. In fact one needs much smaller scale for $\mu_{\text {soft }}$ than the scale $M$. Models with Scaling Ansatz or Simple Real Scaling (SRS) originally proposed in ref. [50] and then analysed at length in refs. [51-57], also belong to the $\mu \tau$ mixing category. As derived in ref. [52], the residual symmetry for SRS is given by

$$
G_{3}^{k}=\left(\begin{array}{ccc}
-1 & 0 & 0 \\
0 & \left(1-k^{2}\right)\left(1+k^{2}\right)^{-1} & 2 k\left(1+k^{2}\right)^{-1} \\
0 & 2 k\left(1+k^{2}\right)^{-1} & -\left(1-k^{2}\right)\left(1+k^{2}\right)^{-1}
\end{array}\right),
$$

where ' $k$ ' is the scale parameter of the model that scales, for example, one row of $M_{\nu}$ with the other row or one column with another column [50]. Thus the scale parameter of the model could be constrained simply by the relation

$$
k=\frac{1 \pm \cos 2 \theta_{g}}{\sin 2 \theta_{g}}
$$

along with eq. (2.7) or eq. (2.12). One can also constrain the parameters of models like four zero textures (in charge lepton flavour basis) within Type-I seesaw [58], so called discrete Dark Matter (DM) models [86, 87] as well as the models with global U(1) symmetries [8891] which at the leading order, show up a $\mu \tau$ mixing scheme at low energy. However, we would like to stress that at this stage, where the precise values of $\delta$ and $\theta_{23}$ are yet to be measured, if the $\mu \tau$ mixing parameter can be constrained a priori by some other constraints, (e.g., the mass-squared differences, the mixing angles other than $\theta_{23}$ or some cosmological phenomenon such as leptogenesis etc.) then eq. (2.7) or eq. (2.12) could be used to predict $\delta$ or $\theta_{23}$. So the models with $\mu \tau$ mixing symmetry and lesser number of parameters (such as Scaling Ansatz+ texture zeros [56]) are most welcome.

\section{$3 \quad \mathrm{CP}$ extension of the $\mu \tau$ mixing symmetry}

\subsection{CP symmetry in a general light neutrino mass term}

So far the discussion was quite general. In this section we want to explore some special class of $\mu \tau$ mixing. Since in general, only flavour symmetries are not sufficient to predict the CP violating phases, a lot of effort has been devoted in past few years to ameliorate flavour symmetries with CP symmetries [77] by demanding the invariance of the neutrino mass term with the field transformation

$$
\nu_{L \ell} \rightarrow i\left(\mathcal{G}_{a}\right)_{\ell m} \gamma^{0} \nu_{L m}^{C} \quad(a=1,2,3) .
$$


Though one always has to respect the 'consistency condition' to have a combined theory of flavour and CP $[65,66]$. The consistency condition can be written as

$$
X_{r} \rho_{r}^{*}(g) X_{r}^{-1}=\rho_{r}\left(g^{\prime}\right),
$$

where $X_{r}$ is a unitary matrix representing CP symmetry which acts on a generic multiplet $\varphi$ as

$$
\varphi(x) \stackrel{\mathrm{CP}}{\longrightarrow} X_{r} \varphi^{*}\left(x^{\prime}\right)
$$

with $x^{\prime}=(t,-\mathbf{x})$ and $\rho_{r}(g), \rho_{r}\left(g^{\prime}\right)$ are the representations for the elements $g, g^{\prime}$ of the flavour group in an irreducible representation $\mathbf{r}$. Eq. (3.1) leads to the complex invariance

$$
\mathcal{G}_{a}^{T} M_{\nu} \mathcal{G}_{a}=M_{\nu}^{*}
$$

Now at low energy, among the three residual $\mathbb{Z}_{2}$ generators, if two of them, say, $\mathcal{G}_{2}$ and $\mathcal{G}_{3}$ correspond to the complex invariances

$$
\mathcal{G}_{2}^{T} M_{\nu} \mathcal{G}_{2}=M_{\nu}^{*}, \mathcal{G}_{3}^{T} M_{\nu} \mathcal{G}_{3}=M_{\nu}^{*}
$$

the remaining one, i.e., $\mathcal{G}_{1}$ automatically satisfies a real invariance [64]

$$
\mathcal{G}_{1}^{T} M_{\nu} \mathcal{G}_{1}=M_{\nu}
$$

It is trivial now to show, that eq. (3.2) is satisfied. Since in this case, the CP and flavour symmetry generators are basically $\mathbb{Z}_{2}$ generators, eq. (3.2) would imply

$$
\mathcal{G}_{2} \mathcal{G}_{1} \mathcal{G}_{2}^{-1}=\mathcal{G}_{1}, \mathcal{G}_{3} \mathcal{G}_{1} \mathcal{G}_{3}^{-1}=\mathcal{G}_{1} .
$$

Since by construction, $\mathcal{G}_{a} \mathcal{G}_{b}=\mathcal{G}_{b} \mathcal{G}_{a}=\mathcal{G}_{c}$ for $a \neq b \neq c$, the left and right hand sides of the above equations are consistent. Similarly, one obtains a real invariance for $\mathcal{G}_{2}$, for the simultaneous complex invariances for $\mathcal{G}_{1}$ and $\mathcal{G}_{3}$. However, note that, if we demand the complex invariances for $\mathcal{G}_{1}$ and $\mathcal{G}_{2}$, we obtain a real invariance for $\mathcal{G}_{3}$ which is not acceptable, since, that will correspond to a vanishing $\theta_{13}$. Let us now turn into the computation of the Dirac CP phase for both the acceptable real invariances, i.e., $\left(\mathcal{G}_{1}^{g \mu \tau}\right)^{T} M_{\nu} \mathcal{G}_{1}^{g \mu \tau}=M_{\nu}$ and $\left(\mathcal{G}_{2}^{g \mu \tau}\right)^{T} M_{\nu} \mathcal{G}_{2}^{g \mu \tau}=M_{\nu}$. For both the cases, the second of (3.5) leads to [92, 93]

$$
\sin \delta= \pm \frac{\sin 2 \theta_{g}}{\sin 2 \theta_{23}}
$$

Eliminating $\theta_{g}$ from (2.7) and (3.8) for $\mathcal{G}_{1}^{g \mu \tau}$ whereas doing the same from (2.12) and (3.8) for $\mathcal{G}_{2}^{g \mu \tau}$ we obtain a generic expression for $\cos \delta$ as

$$
\cos \delta=\frac{A_{i} B \pm \sqrt{A_{i}^{2} B^{2}-\left(B^{2}-C_{i}^{2} \sin ^{2} 2 \theta_{23}\right)\left(A_{i}^{2}-C_{i}^{2} \cos ^{2} 2 \theta_{23}\right)}}{\left(B^{2}-C_{i}^{2} \sin ^{2} 2 \theta_{23}\right)},
$$

where $i=1,2$ corresponds $\mathcal{G}_{i}^{g \mu \tau}$ symmetries. The parameters $A_{i}, B$ and $C_{i}$ are the functions of the mixing angles $\theta_{i j}$ with the explicit expressions

$$
\begin{aligned}
A_{1} & =\left(s_{23}^{2}-c_{23}^{2}\right)\left(s_{12}^{2}-c_{12}^{2} s_{13}^{2}\right), & & A_{2}=\left(c_{23}^{2}-s_{23}^{2}\right)\left(c_{12}^{2}-s_{12}^{2} s_{13}^{2}\right), \\
C_{1} & =\left(s_{13}^{2}+c_{13}^{2} s_{12}^{2}\right), & & C_{2}=-\left(s_{13}^{2}+c_{13}^{2} c_{12}^{2}\right), \\
B & =4 c_{12} s_{12} c_{23} s_{23} s_{13} . & &
\end{aligned}
$$



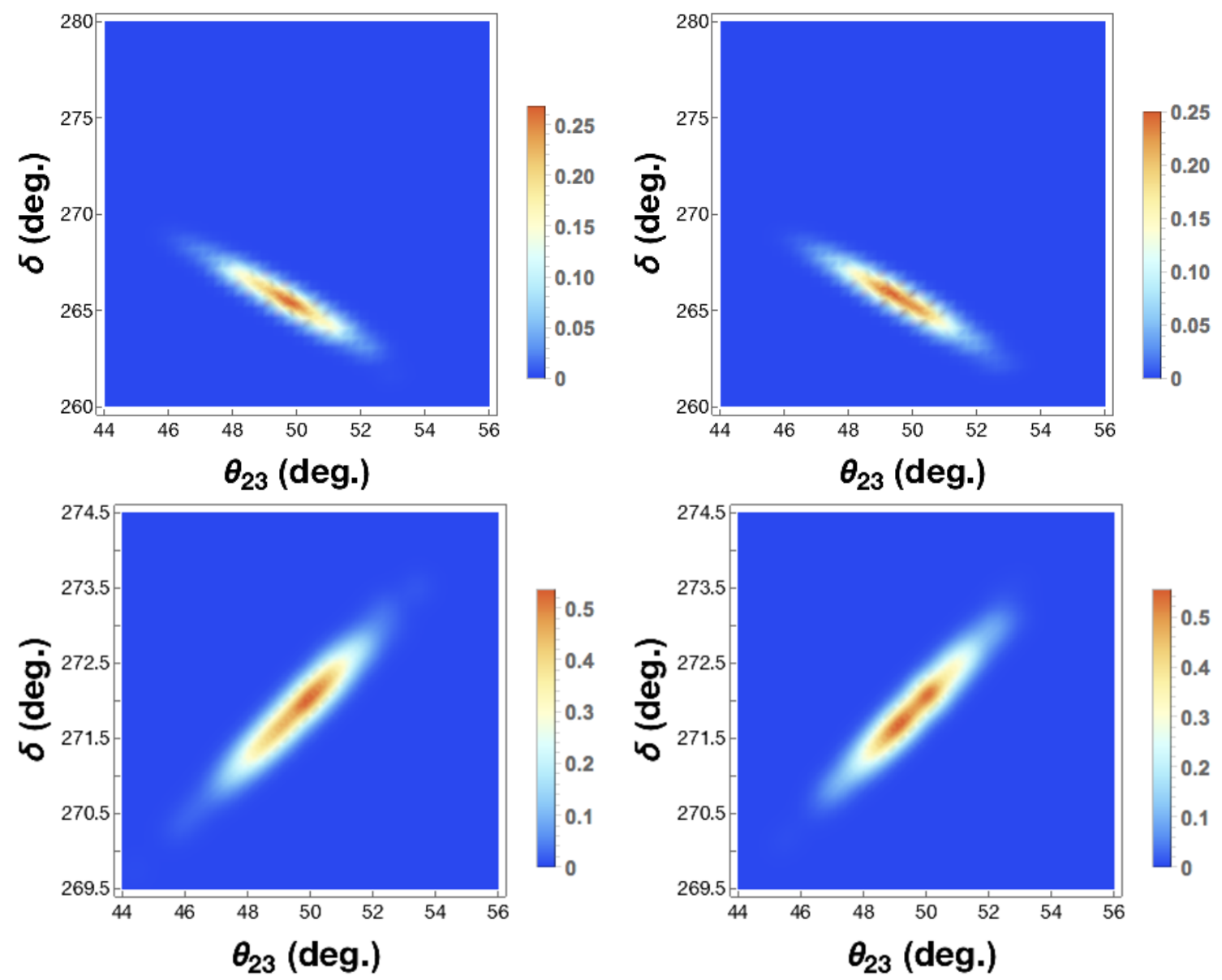

Figure 5. For the real invariance $\left(\mathcal{G}_{1}^{g \mu \tau}\right)^{T} M_{\nu} \mathcal{G}_{1}^{g \mu \tau}=M_{\nu}$ (top): probability distribution of $\delta$ with $\theta_{23}$. For the real invariance $\left(\mathcal{G}_{2}^{g \mu \tau}\right)^{T} M_{\nu} \mathcal{G}_{2}^{g \mu \tau}=M_{\nu}$ (bottom): probability distribution of $\delta$ with $\theta_{23}$. Here we have used Gaussian distribution for each of the mixing angles.

The novel correlations obtained in eq. (3.9) are exact and can be further simplified if terms $\mathcal{O}\left(s_{13}^{4}\right)$ are dropped. Interestingly, both the relations are independent of $\theta_{g}$ and coincide with the prediction $\cos \delta=0$ for $\mathrm{CP}$ extended $\mu \tau\left(\mathrm{CP}^{\mu \tau}\right)[28,29]$ in the limit $\theta_{23} \rightarrow \pi / 4$.

In figure 5 , we show the predictions of $\mathrm{CP}$ extended $\mu \tau$ mixing. The figures in the top panel are for the real invariance for $\mathcal{G}_{1}^{g \mu \tau}$ (for each case there are two solutions due the ' \pm ' sign in eq. (3.9)) and those which are in the bottom panel are for the real invariance due to $\mathcal{G}_{2}^{g \mu \tau}$. Notice that unlike the $\mathrm{CP}^{\mu \tau}$ (CP extended $\mu \tau$ interchange [30]) which predicts co-bimaximal mixing $\left(\delta= \pm 3 \pi / 2\right.$ and $\left.\theta_{23}=\pi / 4\right)$ here nonmaximal atmospheric mixing is allowed, however, the most probable values of $\delta$ are clustered around their near maximal values. Thus significant deviation from maximality of $\delta$ would rule out both the scenarios (present data on $\delta$ for NMO is at $\sim 1.37 \sigma$ tension with the predictions obtained in the CP extended $\mu \tau$ mixing). Nevertheless, we should stress that since both the real invariances lead to almost same predictions on $\delta$ (a preference for near maximality), it is difficult to disentangle these two cases experimentally, unless we look for precision measurements of $\delta$ in 
near future or we do explicit mass models which can be testable through other parameters, e.g, through predictions on masses or neutrino-less double beta decay parameters etc. Before proceeding further into the discussion of the CP extended $\mu \tau$ mixing in Type-I seesaw framework with the motivation to explore possible implications on baryogenesis via leptogenesis, let's point out an interesting aspect regarding the class of the $\mathrm{CP}$ extended $\mu \tau$ mixing within the proposed $\mu \tau$ mixing. We have seen in section 2 , introduction of $\mu \tau$ mixing instead of the $\mu \tau$ interchange symmetry, adds up two more degrees of freedom. To be precise, for both the cases, whilst simultaneous nonmaximal values for $\delta$ and $\theta_{23}$ are allowed, only for the $\mu \tau$ mixing scenario, we can test nonmaximality in one parameter for a maximal value of the other. Therefore there could be two prominent options to look for i) $\delta$ could be maximal but $\theta_{23}$ is not ii) $\theta_{23}$ could be maximal but $\delta$ is not. From figure 5 , it is evident that the $\mathrm{CP}$ extended $\mu \tau$ mixing belongs (approximately) to the case ' $\mathrm{i}$ '. Explicit flavour models for the case 'ii' would also be interesting in future since we don't have any precise statements on the value of $\delta$ and $\theta_{23}$ at this moment.

\subsection{CP symmetry in Type-I seesaw}

We may now proceed to the discussion of this extended CP in Type-I seesaw mechanism. It is well known that to obtain light neutrino masses one has to introduce singlet right handed $(\mathrm{RH})$ fields as a minimal extension to the Standard Model (SM). Thus with the introduction of the singlet fields $N_{R i}$, in the diagonal basis of the RH neutrinos, the Lagrangian for the Type-I seesaw reads

$$
-\mathcal{L}_{\text {mass }}^{\nu}=\bar{N}_{i R}\left(m_{D}\right)_{i \alpha} \nu_{L \alpha}+\frac{1}{2} \bar{N}_{i R}\left(M_{R}\right)_{i} \delta_{i j} N_{j R}^{C}+\text { h.c. }
$$

with $\alpha=e, \mu, \tau$ and $i=1,2,3$. The first term in eq. (3.13) is a Dirac type and the second term is a Majorana type mass term which together lead to the effective $3 \times 3$ light neutrino Majorana mass matrix $M_{\nu}$ as

$$
M_{\nu}=-m_{D}^{T} M_{R}^{-1} m_{D}
$$

In the diagonal basis of the charged lepton as well as the heavy $\mathrm{RH}$ neutrinos, a $\mathrm{CP}$ invariant light neutrino mass matrix

$$
\mathcal{G}^{T} M_{\nu} \mathcal{G}=M_{\nu}^{*}
$$

could be obtained with the following transformation ${ }^{4}$ on $m_{D}$.

$$
m_{D} \mathcal{G}=-m_{D}^{*}
$$

We refer to refs. [30, 55, 94], to realize how in the diagonal basis of charged lepton and heavy neutrinos, CP is applied to the Type-I seesaw Lagrangian. Now in our case, to have a real invariance for $\mathcal{G}_{1}^{g \mu \tau}$ as well as $\mathcal{G}_{2}^{g \mu \tau}$ one needs the following transformations.

$$
m_{D} \mathcal{G}_{i}^{g \mu \tau}=-m_{D}^{*}, m_{D} \mathcal{G}_{3}^{g \mu \tau}=-m_{D}^{*} . \quad(i=1,2)
$$

\footnotetext{
${ }^{4}$ We choose the minus sign in the r.h.s. of eq. (3.16), just to present entries of the first column of 3.18 as real elements. Otherwise, given the form $\mathcal{G}_{3}^{g \mu \tau}$ in eq. (1.6), those elements would be pure imaginary quantities. However, this is just a matter of convention. A plus sign is also equally acceptable.
} 
For both the cases, the most general form of $m_{D}$ that satisfies the second constraint of eq. (3.17) can be parametrized as

$$
m_{D}=\left(\begin{array}{ccc}
a & b_{1}+i b_{2} & -b_{1} \tan \theta_{g}+i b_{2} \cot \theta_{g} \\
e & c_{1}+i c_{2} & -c_{1} \tan \theta_{g}+i c_{2} \cot \theta_{g} \\
f & d_{1}+i d_{2} & -d_{1} \tan \theta_{g}+i d_{2} \cot \theta_{g}
\end{array}\right),
$$

where all the parameters are real and a priori unknown. There will be other constraints (the parameters $b_{1}, c_{1}, d_{1}$ could be expressed in terms $a, e, f$ and $\theta_{g}$ ) on the mass matrix $m_{D}$ of eq. (3.18) due to the first transformation in eq. (3.17). However, those transformations are not important in this paper, since we present the discussion of leptogenesis with few benchmark values (particularly for the decay parameters $K_{i \alpha}$ and the CP asymmetry parameters $\epsilon_{i \alpha}$ as given in the next section) which are always compatible with those transformations. ${ }^{5}$ In any case, those constraint equations could easily be derived as shown in ref. [55]. But what matters here, the overall structure of $m_{D}$ shown in eq. (3.18). Having set up all the necessary prerequisites, we are now ready to explore the baryogenesis via leptogenesis in the $\mathrm{CP}$ extended $\mu \tau$ mixing framework.

\section{Baryogenesis via leptogenesis in the CP extended framework}

Baryogenesis via leptogenesis is a process where $\mathrm{CP}$ violating and out of equilibrium decays of the heavy RH neutrinos produce lepton asymmetry which is thereafter converted to baryon asymmetry by non-perturbative sphalerons [95]. For a simplified discussion we opt for a two RH neutrino scenario where the decays and interactions due to $N_{1}$ and $N_{2}$ would matter to the process of leptogenesis. However, the qualitative results drawn in a two RH neutrino scenario would also be relevant for a three RH neutrino case. When the masses of the two $\mathrm{RH}$ neutrinos are in the regime $M_{i}>10^{12} \mathrm{GeV}$ where all the charged lepton flavours are out of equilibrium [78-80], quantum states $\left|\ell_{i}\right\rangle$ produced by the decay of $\mathrm{RH}$ neutrinos can be written as a coherent superposition of the flavour states $\left|\ell_{\alpha}\right\rangle$ as

$$
\begin{aligned}
& \left|\ell_{i}\right\rangle=\mathcal{A}_{i \alpha}\left|\ell_{\alpha}\right\rangle, \quad \\
& \left|\bar{\ell}_{i}\right\rangle=\overline{\mathcal{A}}_{i \alpha}\left|\bar{\ell}_{\alpha}\right\rangle . \quad(i=1,2, \alpha=e, \mu, \tau)
\end{aligned}
$$

The amplitudes at the tree level are given by

$$
\mathcal{A}_{i \alpha}^{0}=\frac{m_{D_{i \alpha}}}{\sqrt{\left(m_{D} m_{D}^{\dagger}\right)_{i i}}} \quad \text { and } \quad \overline{\mathcal{A}}_{i \alpha}^{0}=\frac{m_{D_{i \alpha}}^{*}}{\sqrt{\left(m_{D} m_{D}^{\dagger}\right)_{i i}}}
$$

Since there is no interaction to break the coherence of the quantum states before it inversely decays to $N_{i}$, the asymmetry will be produced along the direction of $\left|\ell_{i}\right\rangle\left(\right.$ or $\left.\left|\bar{\ell}_{i}\right\rangle\right)$

\footnotetext{
${ }^{5}$ Even after considering the constraints from $\mathcal{G}_{1,2}^{g \mu \tau}$, the number of effective parameters in $m_{D}$ are more than the number of experimental constraints.
} 
in the lepton flavour space. In that case, the set of classical kinetic equations relevant for leptogenesis could be written as [15]

$$
\begin{aligned}
\frac{d N_{N_{i}}}{d z} & =-D_{i}\left(N_{N_{i}}-N_{N_{i}}^{\mathrm{eq}}\right), \text { with } i=1,2 \\
\frac{d N_{B-L}}{d z} & =-\sum_{i=1}^{2} \varepsilon_{i} D_{i}\left(N_{N_{i}}-N_{N_{i}}^{\mathrm{eq}}\right)-\sum_{i=1}^{2} W_{i} N_{B-L},
\end{aligned}
$$

with $z=M_{1} / T$. Eq. (4.4) tracks the dynamics of the RH neutrinos (production+decay) while eq. (4.5) tracks the lepton asymmetry which survives in the interplay of the production (first term) and washout (second term), as a function of $z . \quad N_{N_{i}}$ 's and $N_{B-L}$ are the abundances computed per number of $N_{i}$ 's in ultra-relativistic thermal equilibrium. Defining $x_{i j}=M_{j}^{2} / M_{i}^{2}$ and $z_{i}=z \sqrt{x_{1 i}}$, the decay terms can be written as

$$
D_{i}=\frac{\Gamma_{D, i}}{H z}=K_{i} x_{1 i} z\left\langle 1 / \gamma_{i}\right\rangle,
$$

where total decay rates $\Gamma_{D, i}$ are given by $\Gamma_{D, i}=\bar{\Gamma}_{i}+\Gamma_{i}=\Gamma_{D, i}(T=0)\left\langle 1 / \gamma_{i}\right\rangle$ with $\left\langle 1 / \gamma_{i}\right\rangle$ as the thermally averaged dilation factor and can be expresses as the ratio of two modified Bessel functions as

$$
\left\langle 1 / \gamma_{i}\right\rangle=\frac{\mathcal{K}_{1}\left(z_{i}\right)}{\mathcal{K}_{2}\left(z_{i}\right)}
$$

The decay parameter $K_{i}$ is given by

$$
K_{i} \equiv \Gamma_{D, i}(T=0) / H\left(T=M_{i}\right) .
$$

The equilibrium abundance of $N_{i}$ is given by $N_{N_{i}}^{\mathrm{eq}}=\frac{1}{2} z_{i}^{2} \mathcal{K}_{2}\left(z_{i}\right)$ and the CP asymmetry $\varepsilon_{i}=\sum_{\alpha} \varepsilon_{i \alpha}$ is given by

$$
\varepsilon_{i}=\sum_{\alpha} \frac{\Gamma_{i \alpha}-\bar{\Gamma}_{i \alpha}}{\Gamma_{i}+\bar{\Gamma}_{i}}
$$

with the flavoured $\mathrm{CP}$ asymmetry $\varepsilon_{i \alpha}$ as [96]

$$
\begin{aligned}
\varepsilon_{i \alpha}= & \frac{1}{4 \pi v^{2} h_{i i}} \sum_{j \neq i} \operatorname{Im}\left\{h_{i j}\left(m_{D}\right)_{i \alpha}\left(m_{D}^{*}\right)_{j \alpha}\right\}\left[f\left(x_{i j}\right)+\frac{\sqrt{x_{i j}}\left(1-x_{i j}\right)}{\left(1-x_{i j}\right)^{2}+h_{j j}^{2}\left(16 \pi^{2} v^{4}\right)^{-1}}\right] \\
& +\frac{1}{4 \pi v^{2} h_{i i}} \sum_{j \neq i} \frac{\left(1-x_{i j}\right) \operatorname{Im}\left\{h_{j i}\left(m_{D}\right)_{i \alpha}\left(m_{D}^{*}\right)_{j \alpha}\right\}}{\left(1-x_{i j}\right)^{2}+h_{j j}^{2}\left(16 \pi^{2} v^{4}\right)^{-1}}
\end{aligned}
$$

where $h_{i j} \equiv\left(m_{D} m_{D}^{\dagger}\right)_{i j}$. The final $B-L$ asymmetry could be written as

$$
N_{B-L}^{f}=N_{B-L}^{\text {in }} e^{-\sum_{i} \int d z^{\prime} W_{i}\left(z^{\prime}\right)}+N_{B-L}^{\text {lepto }},
$$

where $N_{B-L}^{\mathrm{in}}$ could be a possible pre-existing asymmetry at an initial temperature $T_{\mathrm{in}}$ and $N_{B-L}^{\text {lepto }}$ is the contribution from pure leptogenesis. In this work we assume any pre-existing asymmetry (so called strong thermal condition [8]) is strongly washed out by the heavy $\mathrm{RH}$ neutrinos. Therefore, we are in a strong washout scenario. Thus in the washout term 
in eq. (4.5), the $\Delta L=1$ scattering term $W_{i}^{\Delta L=1}$ can be safely neglected [79]. However, a particular washout regime is a matter of choice in our discussion. One can neglect the pre-existing asymmetry assuming there is no source of asymmetry production prior to the leptogenesis phase and explore a weak washout regime as well. In that case inclusion of scattering would only affect the asymmetry production efficiency [79] but the qualitative conclusion drawn in a strong washout regime would remain the same. For our purpose, we shall also neglect the non-resonant part of the $\Delta L=2$ term $W_{i}^{\Delta L=2}$ which is relevant only at higher temperature. Now the relevant washout term $W_{i} \simeq W_{i}^{\mathrm{ID}}$ can be written as (after properly subtracting the real intermediate state contribution of $\Delta L=2$ process)

$$
W_{i}^{\mathrm{ID}}=\frac{1}{4} K_{i} \sqrt{x_{1 i}} \mathcal{K}_{1}\left(z_{i}\right) z_{i}^{3} .
$$

The final baryon to photon ratio is given by

$$
\eta_{B}=a_{\mathrm{sph}} \frac{N_{B-L}^{\text {lepto }}}{N_{\gamma}^{\text {rec }}} \simeq 0.96 \times 10^{-2} N_{B-L}^{\text {lepto }},
$$

where $N_{\gamma}^{\text {rec }}$ is the photon density at the recombination and the sphaleron conversion coefficient $a_{\mathrm{sph}} \sim 1 / 3$. In a given model, this $\eta_{B}$ has to be compared with measured value [97]

$$
\eta_{B}^{\mathrm{CMB}}=(6.3 \pm 0.3) \times 10^{-10} .
$$

In the mass regime $10^{9} \mathrm{GeV}<M_{i}<10^{12} \mathrm{GeV}$, interactions due to tau charged lepton flavour are fast enough to break the coherent evolution of the quantum states $\left|\ell_{i}\right\rangle$ before it inversely decays to $N_{i}$. The $\left|\ell_{i}\right\rangle$ is then projected into a two flavour basis characterised by the eigenstates along the directions of $\tau$ and $\tau_{i}^{\perp}=e+\mu$. In the three flavour regime, i.e. $M_{i}<10^{9} \mathrm{GeV}$, the muon charge lepton flavour comes into equilibrium. It breaks the coherent evolution of the states which is along $\tau_{i}^{\perp}$ and one resolves all the flavours $(e, \mu, \tau)$ individually (for both the flavour regimes, we are assuming strong decoherence so that the density matrix ${ }^{6}$ is flavour diagonal $\left.[78,98]\right)$. Thus for each flavour regime, one has to track the lepton asymmetry in the relevant flavours. For example, if we are in the two flavour regime, the lepton asymmetry has to be tracked in $\tau$ and $\tau_{i}^{\perp}$ flavours. The Boltzmann equations for a generic flavour ' $\alpha$ ' could be written as

$$
\begin{aligned}
& \frac{d N_{N_{i}}}{d z}=-D_{i}\left(N_{N_{i}}-N_{N_{i}}^{\mathrm{eq}}\right), \text { with } i=1,2 \\
& \frac{d N_{\Delta_{\alpha}}}{d z}=-\sum_{i=1}^{2} \varepsilon_{i \alpha} D_{i}\left(N_{N_{i}}-N_{N_{i}}^{\mathrm{eq}}\right)-\sum_{i=1}^{2} P_{i \alpha}^{0} W_{i}^{\mathrm{ID}} N_{\Delta_{\alpha}} .
\end{aligned}
$$

Here $N_{\Delta_{\alpha}}$ is the asymmetry in the flavour $\alpha$ analytic solution for which can be obtained as

$$
N_{\Delta_{\alpha}}=-\sum_{i}^{2} \varepsilon_{i \alpha} \kappa_{i \alpha}
$$

\footnotetext{
${ }^{6}$ An elaborate computation of leptogenesis in density matrix formalism is given in ref. [99].
} 
with the efficiency factor

$$
\kappa_{i \alpha}(z)=-\int_{z_{\text {in }}}^{\infty} \frac{d N_{N_{i}}}{d z^{\prime}} e^{-\sum_{i} \int_{z^{\prime}}^{z} P_{i \alpha}^{0} W_{i}^{\mathrm{ID}}\left(z^{\prime \prime}\right) d z^{\prime \prime}} d z^{\prime} .
$$

For numerical integration purposes one can set very small value of ' $z_{\text {in }}(\sim 0)$ ' and a very large value for ' $z\left(\sim 10^{3}\right)$ '. The final baryon to photon ratio is then given by

$$
\eta_{B}=0.96 \times 10^{-2} \sum_{\alpha} N_{\Delta_{\alpha}}
$$

The quantity $P_{i \alpha}^{0}$ is the tree level probability of a quantum state produced by the $i$ th heavy neutrino being in the flavour $\alpha$ and has an expression

$$
P_{i \alpha}^{0} \equiv K_{i \alpha} / K_{i}
$$

where $K_{i \alpha}$ is the flavoured decay parameter defined as

$$
K_{i \alpha}=\frac{\Gamma_{i \alpha}+\bar{\Gamma}_{i \alpha}}{H\left(T=M_{i}\right)} \equiv \frac{\left|m_{D_{i \alpha}}\right|^{2}}{M_{i} m^{*}}
$$

with $m^{*} \simeq 10^{-3}$ being the equilibrium neutrino mass. Let us mention another important parameter $\Delta P_{i \alpha}=P_{i \alpha}-\bar{P}_{i \alpha}$ strongly relevant to our discussion. The tree+loop level projectors are given by $P_{i \alpha}=\left|\mathcal{A}_{i \alpha}\right|^{2}=P_{i \alpha}^{0}+\frac{\Delta P_{i \alpha}}{2}, \bar{P}_{i \alpha}=\left|\overline{\mathcal{A}}_{i \alpha}\right|^{2}=P_{i \alpha}^{0}-\frac{\Delta P_{i \alpha}}{2}$. The quantity $\Delta P_{i \alpha}$, the difference between the tree+loop level projectors, is nonzero since, in general $\left|\mathcal{A}_{i \alpha}\right| \neq$ $\left|\overline{\mathcal{A}}_{i \alpha}\right|$ [13]. Now the flavoured CP asymmetry parameter $\varepsilon_{i \alpha}$ of eq. (4.9) can be simplified as

$$
\varepsilon_{i \alpha}=P_{i \alpha}^{0} \varepsilon_{i}+\Delta P_{i \alpha} / 2 .
$$

Though the quantity $\Delta P_{i \alpha}$ is not significant in the washout terms, for the CP asymmetry parameter it is remarkably relevant. In fact we show in the model under consideration, the entire source of CP violation in a particular flavour ' $\alpha$ ' arises due to the $\Delta P_{i \alpha}$ term.

As mentioned in the introduction, here we discuss only the three flavour regime $\left(M_{i}<\right.$ $10^{9} \mathrm{GeV}$ ) of the leptogenesis to show the dramatic difference between the conclusion drawn in case of a $\mathrm{CP}$ extended $\mu \tau$ interchange $[30,32,94]$ and the proposed $\mathrm{CP}$ extended $\mu \tau$ mixing. Let's clarify explicitly why we do that.

One flavour regime. First of all, for the one flavour regime $\left(M_{i}>10^{12} \mathrm{GeV}\right)$, the second term in eq. (4.10) is vanishing when summed over ' $\alpha$ ', i.e, $\operatorname{Im}\left\{h_{j i}\left(m_{D}\right)_{i \alpha}\left(m_{D}^{*}\right)_{j \alpha}\right\}=$ $\operatorname{Im}\left[\left|h_{j i}\right|^{2}\right]=0$. The first term is proportional to $\operatorname{Im}\left\{h_{i j}^{2}\right\}$. Using eq. (3.18), the ' $h=$ $m_{D} m_{D}^{\dagger}$ ' can be shown to be a real matrix. Thus the flavour summed CP asymmetry $\varepsilon_{i}=\sum_{\alpha} \varepsilon_{i \alpha}$ vanishes for any ' $i$ '. Therefore successful baryogenesis is not possible in the unflavoured regime. This result has also been obtained in CP extended $\mu \tau$ interchange symmetry $[30,32,94]$. One can also show $\varepsilon_{i e}=0$, since the first column of the $m_{D}$ matrix in eq. (3.18) is real. Thus similar to CP extended $\mu \tau$ interchange, $\varepsilon_{i \mu} \equiv \Delta P_{i \mu} / 2=-\varepsilon_{i \tau}$. Therefore, in the one flavour regime, the results obtained for leptogenesis in $\mathrm{CP}$ extended $\mu \tau$ mixing, are similar to $\mathrm{CP}$ extended $\mu \tau$ interchange. 
Two flavour regime. As already mentioned, we are probing a strong washout scenario assuming any pre-existing asymmetry produced prior to leptogenesis phase has been strongly washed out. This is only possible in the three flavour regime. For the two flavour regime $\left(10^{9} \mathrm{GeV}<M_{i}<10^{12} \mathrm{GeV}\right)$, note that this is not possible since, though in the direction of $\tau$ flavour the asymmetry could be washed out assuming $K_{i \tau} \gg 1$, however, a component of the asymmetry would always survive in the direction orthogonal to the $\tau_{\perp}[98,100]$, irrespective of the value of $K_{\tau_{\perp}}$. Thus a pure leptogenesis scenario breaks down. In any case, as mentioned earlier, along with the strong washout scenario, one can also probe the weak washout regime relaxing the strong-thermal condition (pre-existing asymmetry), which has been done so far in the literature in the context of $\mathrm{CP}^{\mu \tau}$ ). However, for the latter case, apart form showing a successful baryogenesis, we hardly expect any prediction on low energy neutrino parameters, since the number of parameters in $m_{D}$ is still larger than the number of experimental constraints. Thus in the two flavour regime, from leptogenesis perspective, there will be no significant difference between a CP extended $\mu \tau$ interchange and a $\mathrm{CP}$ extended $\mu \tau$ mixing. But certainly differences will be there if one assumes texture zeros on top of the $\mathrm{CP}$ extended $\mu \tau$ mixing $[58,73,101]$ or imposes the symmetry in a minimal seesaw framework $[32,101]$. Since in that case there would be less number of parameters and one might expect predictions from the baryogenesis constraint on the physical parameters such as $\theta_{23}$ which is nonmaximal in general in the $\mu \tau$ mixing scheme. However, an elaborate description in this context, is beyond the scope of this paper.

Three flavour regime. Now coming back to the discussion of leptogenesis in the three flavour regime, first of all one has to go beyond the hierarchical scenario, since in the hierarchical limit the $\mathrm{CP}$ asymmetry parameter, say, $\varepsilon_{1 \alpha}$ is proportional to $M_{1}$ and if $M_{1}<10^{9} \mathrm{GeV}$, one can not generate the observed baryon asymmetry [14]. However if the mass differences of the $\mathrm{RH}$ neutrinos are close enough, one expects a significant enhancement in the loop functions, particularly self energy contribution to the CP asymmetry parameter increases and therefore even if $M_{i}<10^{9} \mathrm{GeV}$, required baryon asymmetry could be generated due to this enhancement in the CP asymmetry parameter [81]. However, in that scenario one has to consider the asymmetry generated by all the heavy neutrinos, since in the standard hierarchical scenario, contribution from the heavier RH neutrinos are washed out by the lighter RH neutrinos. In the limit of quasi-degeneracy (QD) in the RH neutrino spectrum, the contribution from the heavier neutrinos can not be washed out. For an explicit analytical explanation of leptogenesis due to QD mass spectrum, we refer to [102]. For the CP extended $\mu \tau$ interchange symmetry as well as mixing, the $N_{B-L}$ asymmetry could be written as

$$
N_{B-L}^{f}=\sum_{\alpha} N_{\Delta_{\alpha}}=-\sum_{i}^{2}\left(\varepsilon_{i \tau} \kappa_{i \tau}+\varepsilon_{i \mu} \kappa_{i \mu}\right)=-\sum_{i}^{2} \varepsilon_{i \tau}\left(\kappa_{i \tau}-\kappa_{i \mu}\right)=-\sum_{i}^{2} \varepsilon_{i \tau} \kappa_{i}^{\mathrm{eff}},
$$

where we use the fact that $\varepsilon_{i e}=0$ and $\varepsilon_{i \mu}=-\varepsilon_{i \tau}$ and at $z \rightarrow \infty$, the efficiency factor $\kappa_{i \alpha}$ has the expression

$$
\kappa_{i \alpha}=-\int_{0}^{\infty} \frac{d N_{N_{i}}}{d z^{\prime}} e^{-\sum_{i} \int_{z^{\prime}}^{\infty}\left(K_{i \alpha} / K_{i}\right) W_{i}^{\mathrm{ID}}\left(z^{\prime \prime}\right) d z^{\prime \prime}} d z^{\prime} \quad \alpha=(\tau, \mu)
$$


Now notice that, for $\mathrm{CP}$ extended $\mu \tau$ interchange $\left(\theta_{g} \rightarrow \pi / 4\right)$, using eq. (3.18) and eq. (4.21) the decay parameters can be obtained as

$$
K_{1 \mu}^{I}=\frac{b_{1}^{2}+b_{2}^{2}}{M_{1} m^{*}}=K_{1 \tau}^{I}, \quad K_{2 \mu}^{I}=\frac{c_{1}^{2}+c_{2}^{2}}{M_{2} m^{*}}=K_{2 \tau}^{I},
$$

where ' $I$ ' stands for 'Interchange'. Thus from eq. (4.24) one concludes $\kappa_{i \mu}=\kappa_{i \tau}$ and hence, from eq. (4.23), $N_{B-L}^{f}=0$. Therefore, even if we are in the resonance regime of leptogenesis, baryon asymmetry vanishes due to the exact cancellation of the efficiency factors. But for the $\mathrm{CP}$ extended $\mu \tau$ mixing this is not the case. This is since, though the decay parameters $K_{i \mu}$ have the same expression as shown in eq. (4.25), since $\theta_{g} \neq 0$ in general, $K_{i \tau}^{M}$ can be obtained as

$$
K_{i \tau}^{M}=\frac{\left[\operatorname{Re}\left(m_{D}\right)_{i \mu}\right]^{2} \tan ^{2} \theta_{g}+\left[\operatorname{Im}\left(m_{D}\right)_{i \mu}\right]^{2} \cot ^{2} \theta_{g}}{M_{i} m^{*}}
$$

which reduces to $K_{i \tau}^{I}$ of eq. (4.25) in the limit $\theta_{g} \rightarrow \pi / 4$. Here ' $M$ ' stands for 'Mixing'. In fact, given the distribution of $\delta$ in figure 5 , we can approximate $\sin \delta \sim 1$ and therefore using eq. (3.8), we can recast $K_{i \tau}^{M}$ as

$$
K_{i \tau}^{M}=\frac{\left[\operatorname{Re}\left(m_{D}\right)_{i \mu}\right]^{2} \tan ^{2} \theta_{23}+\left[\operatorname{Im}\left(m_{D}\right)_{i \mu}\right]^{2} \cot ^{2} \theta_{23}}{M_{i} m^{*}} .
$$

Thus since $K_{i \tau}^{M} \neq K_{i \mu}^{M}$, from eq. (4.23) $N_{B-L}$ is nonvanishing. It is now clear that to obtain a nonzero baryon asymmetry, in this CP extended $\mu \tau$ mixing framework, one always needs deviation of $\theta_{23}$ from maximality. Now parametrizing $\theta_{23}$ as $\theta_{23}=\left(\pi / 4+\delta_{x}\right)$, where the parameter $\delta_{x}$ accounts for the nonmaximality of $\theta_{23}$, eq. (4.27) could further be simplified as

$$
K_{i \tau}^{M}=K_{i \tau}^{I}\left(1+4 \delta_{x} \cos 2 \xi_{i}\right), \quad \xi_{i}=\tan ^{-1} \frac{\operatorname{Im}\left[\left(m_{D}\right)_{i \mu}\right]}{\operatorname{Re}\left[\left(m_{D}\right)_{i \mu}\right]}
$$

Thus barring a very special solution $\cos 2 \xi_{i}=0$, one always obtains a nonvanishing baryon asymmetry for a nonmaximal value of $\theta_{23}$. Now the $\kappa_{i}^{\text {eff }}$ of eq. (4.23) can be obtained as

$$
\kappa_{i}^{\mathrm{eff}}=4 \delta_{x} K_{i \tau}^{I} \cos 2 \xi_{i} \int_{0}^{\infty} \frac{d N_{N_{i}}}{d z^{\prime}} e^{-\sum_{i} \int_{z^{\prime}}^{\infty} K_{i \tau}^{I} K_{i}^{-1} W_{i}^{\mathrm{ID}}\left(z^{\prime \prime}\right) d z^{\prime \prime}} \sum_{i} \int_{z^{\prime}}^{\infty} K_{i}^{-1} W_{i}^{\mathrm{ID}}\left(z^{\prime \prime}\right) d z^{\prime \prime} d z^{\prime} .
$$

For convenience we may choose $\cos 2 \xi_{i} \equiv \cos 2 \xi=1^{7}$ and flavoured decay parameters for both the RH neutrinos are the same. Then we may further parametrise the $\kappa_{i}^{\text {eff }}$ as

$$
\kappa_{i}^{\mathrm{eff}} \leq \bar{m}_{\max } \frac{4 \delta_{x}}{m^{*}} \int_{0}^{\infty} \frac{d N_{N_{i}}}{d z^{\prime}} e^{-\sum_{i} \int_{z^{\prime}}^{\infty} K_{i \tau}^{I} K_{i}^{-1} W_{i}^{\mathrm{ID}}\left(z^{\prime \prime}\right) d z^{\prime \prime}} \sum_{i} \int_{z^{\prime}}^{\infty} K_{i}^{-1} W_{i}^{\mathrm{ID}}\left(z^{\prime \prime}\right) d z^{\prime \prime} d z^{\prime},
$$

where $\bar{m}$ is the overall mass scale of the light neutrinos. The effective efficiency factor in eq. (4.30) has very interesting features. First of all, from the perspective of $\kappa_{1}^{\text {eff }}$ (effective

\footnotetext{
${ }^{7}$ This is always not the case. The parameter $\xi$ is model dependent. However, as we have already pointed out earlier, the models with CP symmetries, one can not constrain the mass matrix element only by oscillation data, unless some special conditions are assumed [73]. Thus $\cos 2 \xi=1$ would be a probable solution.
} 
production efficiency due of $N_{1}$ ), it encounters a two step suppression. The first one is due to the $N_{2}$-washout, since unlike the hierarchical scenario, in the QD limit $N_{2}$ washout significantly reduces the asymmetry produced by $N_{1}[102]$ and the second one is due the $\delta_{x}$ which appears as a pre-factor in eq. (4.30). In case of $\kappa_{2}^{\text {eff }}$, firstly it increases since the $N_{1}$ interactions cannot fully washout the asymmetry produced by $N_{2}$ (the production from $N_{2}$ is still on), however again similar to $\kappa_{1}^{\text {eff }}$, it faces a suppression by $\delta_{x}$. However, due to the small mass splitting between the $\mathrm{RH}$ neutrino masses, at the end, one obtains comparable production efficiencies $\left(\kappa_{1}^{\text {eff }} \simeq \kappa_{2}^{\text {eff }}\right)$. Let us now have a numerical estimate of the final baryon asymmetry. From eq. (4.14), it is clear that $N_{B-L}=\sum_{\alpha} N_{\Delta_{\alpha}} \simeq 6.3 \times 10^{-8}$. Now if we choose a very small value of the pre-factor in eq. (4.29), say, $4 \delta_{x} K_{i \tau}^{I} \cos 2 \xi_{i} \simeq 0.1$ (this can be done, e.g., either by choosing a very small value of $\cos 2 \xi_{i}$ or very small value of $\delta_{x}$ ), a numerical integration of eq. (4.29) gives $\kappa_{i}^{\text {eff }} \simeq 2 \times 10^{-5}$, where we have assumed $K_{i \mu}=K_{i \tau}=25$. Therefore, to be consistent with the observed value of $N_{B-L}$, one needs $\left|\varepsilon_{i \tau}\right| \simeq 1.5 \times 10^{-3}$. This has also been reproduced in figure 6 by solving the Boltzmann equations, assuming both the RH neutrinos contribute equally (this is justified when one chooses very small mass splitting between RH neutrinos, which is needed in this scheme to obtain resonance in the three flavour regime). One can also comment on the mass scale of the RH neutrinos. For example, let say the resonant enhancement of the CP asymmetry happens when the mass difference $\Delta=\sqrt{x_{12}}-1 \simeq 10^{-7}$. Then assuming the elements of $m_{D}$ in eq. (4.10) as $m_{D} \sim \sqrt{M \bar{m}}$ one obtains the mass scale of the RH neutrinos $M \sim 10^{7} \mathrm{GeV}$. One can explore another interesting situation, assuming $\cos 2 \xi=1, \bar{m}_{\max } \simeq$ $\sqrt{\left|\Delta m_{23}^{2}\right|}$ and the current best-fit of $\theta_{23} \simeq 49.6^{0}\left(\delta_{x} \sim 4.6^{0}\right)$. This would correspond to the value of the pre-factor in eq. (4.30) as $\bar{m}_{\max } \frac{4 \delta_{x}}{m^{*}} \simeq 8$. In that case the correct value of $\eta_{B}$ could be generated with $\left|\varepsilon_{i \tau}\right| \simeq 2.6 \times 10^{-5}$ and consequently the mass scale of the RH neutrinos could be lowered to $\sim 100 \mathrm{TeV}$. Having established the possibility of resonant leptogenesis in the CP extended $\mu \tau$ mixing scheme, the main purpose of the leptogenesis study in this paper is served. However, still one would like to consider some other interesting possibilities such as flavour coupling [82-84]. So far we have discussed the leptogenesis scenario without flavour coupling matrix [80] in the Boltzmann equations. With flavour couplings the Boltzmann equation of eq. (4.15) and eq. (4.16) will be modified as

$$
\begin{aligned}
\frac{d N_{N_{i}}}{d z} & =-D_{i}\left(N_{N_{i}}-N_{N_{i}}^{\mathrm{eq}}\right), \text { with } i=1,2 \\
\frac{d N_{\Delta_{\alpha}}}{d z} & =-\sum_{i=1}^{2} \varepsilon_{i \alpha} D_{i}\left(N_{N_{i}}-N_{N_{i}}^{\mathrm{eq}}\right)-\sum_{i=1}^{2} P_{i \alpha}^{0} W_{i}^{\mathrm{ID}} \sum_{\beta=e, \mu, \tau} C_{\alpha \beta} N_{\Delta_{\beta}},
\end{aligned}
$$

where the flavour coupling matrix $C_{\alpha \beta}$ is given by

$$
C_{\alpha \beta}=\left(\begin{array}{ccc}
188 / 179 & 32 / 179 & 32 / 179 \\
49 / 358 & 500 / 537 & 142 / 537 \\
49 / 358 & 142 / 537 & 500 / 537
\end{array}\right)
$$

which properly accounts for the asymmetry in lepton doublets as well as Higgs asymmetry. One might wonder whether the flavour coupling effect can save the situation for the CP 


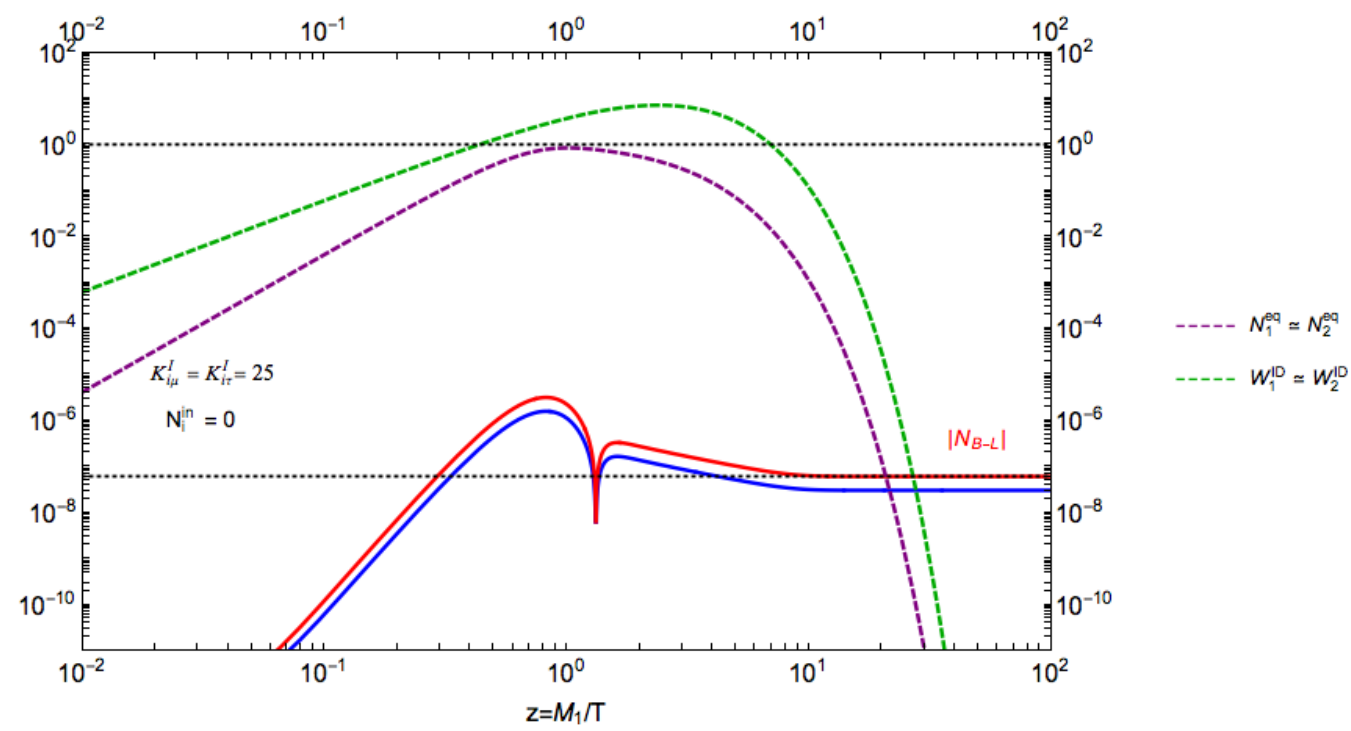

Figure 6. Variation of $N_{B-L}$ with $z$ assuming $4 \delta_{x} K_{i \tau}^{I} \cos 2 \xi=0.1$ and $\left|\varepsilon_{i \tau}\right| \simeq 1.5 \times 10^{-3}$. The blue line is the contribution from a single $\mathrm{RH}$ neutrino. The red line which matches the observed range, represents contributions from both the $\mathrm{RH}$ neutrinos.

extended $\mu \tau$ interchange symmetry, i.e., whether the entries of the flavour coupling can create a mismatch between the decay parameters so that one obtains a nonzero $\kappa_{i}^{\text {eff }}$. Starting from the simplest case, i.e., assuming the diagonal structure of the $C$ matrix (which is usually used in the leptogenesis computations for neutrino mass models), if one writes the Boltzmann equations, the scenario does not change. This is since the $C_{\mu \mu}$ and $C_{\tau \tau}$ elements are the same and therefore they are unable to create any mismatch between the decay parameters. Thus similar to the previous scenario $(C=\mathbb{I})$ there will be no net lepton asymmetry. Interestingly, even if one assumes the nondiagonal structure of the $C$ matrix, one cannot generate nonzero lepton asymmetry. Since, $P_{i \mu}^{0}=P_{i \tau}^{0}$, the muon and tau flavour will couple each other with equal strength $\left(142 P_{i \mu}^{0} / 537\right)$. Therefore there will be no net asymmetry mismatch since we already know the production is equal an opposite $\left(\varepsilon_{i \mu}=-\varepsilon_{i \tau}\right)$. Mathematically, this can be understood in the following way. We can go to a basis where the Boltzmann equation in eq. (4.32) is diagonal in a generic flavour space, say $\alpha^{\prime}$. We can do this by the means of a unitary transformation as

$$
\frac{d N_{\Delta_{\alpha^{\prime}}}}{d z}=-\sum_{i=1}^{2} \varepsilon_{i \alpha^{\prime}} D_{i}\left(N_{N_{i}}-N_{N_{i}}^{\mathrm{eq}}\right)-\sum_{i=1}^{2} W_{i}^{\mathrm{ID}} V^{-1} P_{i \alpha}^{0} C_{\alpha \beta} V N_{\Delta_{\beta^{\prime}}},
$$

where

$$
N_{\Delta_{\beta^{\prime}}}=V^{-1} N_{\Delta_{\beta}}, \quad \varepsilon_{i \alpha^{\prime}}=V^{-1} \varepsilon_{i \alpha}, \quad V^{-1} P_{i \alpha}^{0} C_{\alpha \beta} V=P_{i \beta^{\prime}}^{0} \delta_{\alpha^{\prime} \beta^{\prime}} .
$$

Now similar to eq. (4.17), the $N_{B-L}$ in the prime basis can be written as

$$
N_{B-L}=\sum_{\alpha^{\prime}} N_{\Delta_{\alpha^{\prime}}}=-\sum_{\alpha^{\prime}} \sum_{i}^{2} \varepsilon_{i \alpha^{\prime}} \kappa_{i \alpha^{\prime}}
$$


For numerical purpose, let's assume the total decay parameter $K_{i}=60, K_{i \mu}=K_{i \tau}=25$. Thus the matrix $V$ which diagonalizes $P_{i \alpha}^{0} C_{\alpha \beta}$ is given by

$$
V=\left(\begin{array}{ccc}
0.125 & 0.000 & -0.971 \\
0.701 & -0.707 & 0.166 \\
0.701 & 0.707 & 0.166
\end{array}\right)
$$

This implies

$$
\left(\begin{array}{c}
\varepsilon_{i e^{\prime}} \\
\varepsilon_{i \mu^{\prime}} \\
\varepsilon_{i \tau^{\prime}}
\end{array}\right)=\left(\begin{array}{ccc}
0.125 & 0.000 & -0.971 \\
0.701 & -0.707 & 0.166 \\
0.701 & 0.707 & 0.166 .
\end{array}\right)^{-1}\left(\begin{array}{c}
\varepsilon_{i e} \\
\varepsilon_{i \mu} \\
\varepsilon_{i \tau}
\end{array}\right)=\left(\begin{array}{c}
0 \\
1.414 \varepsilon_{i \tau} \\
0
\end{array}\right)
$$

Therefore the asymmetry vector in the prime basis is given by

$$
\left(\begin{array}{l}
N_{\Delta_{e^{\prime}}} \\
N_{\Delta_{\mu^{\prime}}} \\
N_{\Delta_{\tau^{\prime}}}
\end{array}\right)=\left(\begin{array}{c}
0 \\
-1.414 \sum_{i} \varepsilon_{i \tau} \kappa_{i \mu^{\prime}} \\
0
\end{array}\right)
$$

which should then be transformed in the unprimed basis (original basis of leptogenesis) as

$$
\left(\begin{array}{l}
N_{\Delta_{e}} \\
N_{\Delta_{\mu}} \\
N_{\Delta_{\tau}}
\end{array}\right)=\left(\begin{array}{ccc}
0.125 & 0.000 & -0.971 \\
0.701 & -0.707 & 0.166 \\
0.701 & 0.707 & 0.166 .
\end{array}\right)\left(\begin{array}{c}
0 \\
-1.414 \sum_{i} \varepsilon_{i \tau} \kappa_{i \mu} \\
0
\end{array}\right)=\left(\begin{array}{c}
0 \\
0.996 \sum_{i} \varepsilon_{i \tau} \kappa_{i \mu^{\prime}} \\
-0.996 \sum_{i} \varepsilon_{i \tau} \kappa_{i \mu^{\prime}}
\end{array}\right)
$$

Thus $\sum_{\alpha} N_{\Delta_{\alpha}}=0$ and we have vanishing $N_{B-L}$. However for the proposed $\mu \tau$ mixing scheme, where $P_{i \mu}^{0} \neq P_{i \tau}^{0}$ in general, the asymmetry in the muon and tau flavour will couple each other with different coupling strength. Thus even if we consider diagonal $C$ matrix, we obtain a nonvanishing lepton asymmetry. But most interesting point is, when we take the general form of the $C$ matrix (nondiagonal), a portion of the net asymmetry generated due to the interplay of the muon and tau flavour, will be injected in the electron flavour also. Thus even if we start form a scenario with vanishing production term in the electron flavour $\left(\varepsilon_{i e}=0\right)$ due to the off-diagonal terms mainly due to the $C_{e \mu}$ and $C_{e \tau}$ term we can generate a nonvanishing lepton asymmetry in the electron flavour. Though, for a fixed value of $\varepsilon_{i \alpha}$, magnitude of the injected asymmetry will depend on how strong the mismatch of the asymmetry in muon and tau flavour. In figure 7, along with number densities of the $\mathrm{RH}$ neutrinos and flavoured inverse decay rates (dashed lines), we present the variation of $\left|N_{B-L}\right|$ (solid lines). The blue line (solid) represents the injected asymmetry in the electron flavour. The green (solid) represents the net asymmetry generated by muon and tau flavour. The red (solid) line is the final $N_{B-L}$ when we combine all the flavours. This is clear that after taking into account the asymmetry in electron flavour, we obtain correct value of $N_{B-L}$. This shows the importance of the off-diagonal terms in the flavour coupling matrix which are neglected in general in the computation in leptogenesis. The discussed CP 


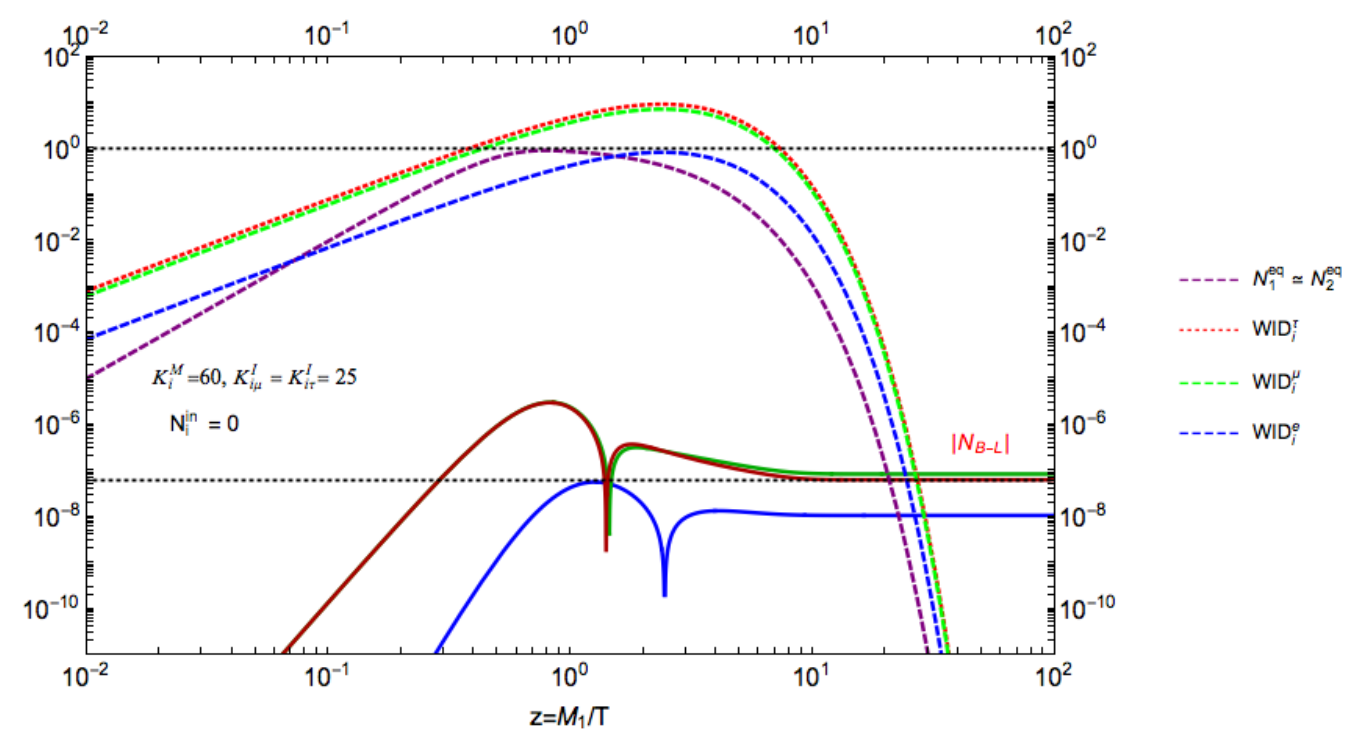

Figure 7. Variation of $N_{B-L}$ with $z$ assuming $\bar{m}_{\max } \frac{4 \delta_{x}}{m^{*}}=8$ and $\left|\varepsilon_{i \tau}\right| \simeq 2.67 \times 10^{-5}$. The blue line is the asymmetry injected in the electron flavour through flavour couplings. The green line is net contribution from the muon and tau flavour asymmetries. The red line which matches the observed range after taking into account the injected asymmetry in the electron flavour.

extended $\mu \tau$ mixing is thus a novel low energy model which facilitates the understanding of flavour couplings in Boltzmann equations for leptogenesis in a very clear way.

Before concluding, we would like to highlight the main results of this paper and make few remarks regarding future prospect of this work.

- We derive model independent correlations between the Dirac CP phase and the light neutrino mixing angles for generalized associate $\mu \tau$ symmetries which we name as the associate $\mu \tau$ mixing symmetries.

- We have shown that the current data on $\delta$ and $\theta_{23}$ could be better explained by the proposed mixing symmetry.

- After a general discussion on $\mu \tau$ mixing which can be realized in many of the neutrino mass models, we discuss the $\mathrm{CP}$ extension of it and find novel testable correlations between $\delta$ and the light neutrino mixing angles.

- We then discuss the baryogenesis via leptogenesis mechanism in the three flavour regime and show unlike the $\mathrm{CP}$ extended $\mu \tau$ interchange, a resonant leptogenesis is possible in the $\mathrm{CP}$ extended $\mu \tau$ mixing and a nonzero baryon to photon ratio always requires nonmaximal $\theta_{23}$ which is now preferred by the current data.

- We have shown quantitatively, even after inclusion of flavour coupling effect in leptogenesis computation, the usually drawn conclusion of a vanishing asymmetry in the fully flavoured regime is still valid for the $\mathrm{CP}$ extended $\mu \tau$ interchange symmetry. 
- The proposed CP extended $\mu \tau$ mixing is a novel example of a neutrino mass model where the role of flavour couplings in leptogenesis mechanism is very explicit.

The paper is presented entirely from mixing perspective since, whilst the $\mu \tau$ mixing or its $\mathrm{CP}$ extensions are very attractive in the light neutrino mixing sector, they don't entertain predictions in the mass sector. Therefore, explicit models with lesser number of parameters with $\mu \tau$ mixing or its $\mathrm{CP}$ extended version would be of great interest to search for. Since in that case, besides all the model independent correlations presented in this work, there would be definite statements on the masses as well. These models would also have interesting consequences in leptogenesis. For example, as we have shown in the CP extended version of $\mu \tau$ mixing, since we don't have the predictions on the masses, we have assumed conditions such as $\cos 2 \xi=1, K_{i \tau}=25$ etc. But in the models with less number of parameters, we would have exact statements on the assumed parameters. In that case we can have precise statement on the nonmaximality of $\theta_{23}$ required to generate the observed baryon asymmetry via resonant leptogenesis mechanism even if considering the RH mass scale at $\mathcal{O}(\mathrm{TeV})[81,103]$. To constrain the parameter space of such predictive models, it would be then interesting to consider the heavy neutrino flavour oscillation effects [104-106] to increase the robustness of the low energy predictions.

\section{Conclusions}

In this work we promote the idea of $\mu \tau$ mixing symmetry which is a generalized version of the $\mu \tau$ interchange symmetry. After systematically deriving the model independent correlations among the Dirac CP phase and the light neutrino mixing angles in the $\mu \tau$ mixing scheme, we show that the stringent condition of simultaneous maximality of $\delta$ and $\theta_{23}$ which is a well known prediction of the interchange symmetry (currently disfavoured by oscillation data) could be relaxed. We show that the present data or the current trend on $\delta$ and $\theta_{23}$ seeks deviation from the $\mu \tau$ interchange scheme. Encoding the deviation in a single parameter $\theta_{g}$ which generalizes $\mu \tau$ interchange to the $\mu \tau$ mixing for $\theta_{g} \neq \pi / 4$, we then comment on the strength of the deviation to be consistent with present data and also show how the parameter $\theta_{g}$ could be related to the model parameters in neutrino mass models that exhibit $\mu \tau$ mixing at the low energy. Inclusion of the parameter $\theta_{g}$ opens up possibilities to explore several classes of the $\mu \tau$ mixing scheme. We particularly discuss the CP extended version of the $\mu \tau$ mixing symmetry and derive novel correlations among $\delta$ and the light neutrino mixing angles. Particularly we show, in this class of models, most probable values of $\delta$ prefers maximal Dirac CP violation while the atmospheric mixing angle $\theta_{23}$ is not necessarily maximal. We also show, that unlike the CP extended version of the $\mu \tau$ interchange, the mixing scenario is able to explain the observed baryon asymmetry in the three flavour regime via resonant leptogenesis mechanism. Particularly we show, barring a very special choice in the parameter space, the observed baryon asymmetry is proportional to the deviation of $\theta_{23}$ from its maximality. Thus to explain baryon asymmetry simultaneously with neutrino mixing, the $\mathrm{CP}$ extended $\mu \tau$ mixing symmetry favours nonmaximal values of $\theta_{23}$. After a qualitative as well as quantitative comparison of the leptogenesis 
scenario in the three flavour regime between the $\mathrm{CP}$ extended interchange and mixing symmetry, we show, whilst for the interchange scenario, even if we include off-diagonal flavour coupling matrix $(C)$ in the Boltzmann equation for the leptonic number densities, the usual conclusion of obtaining a vanishing asymmetry in the three flavour regime is unchanged, however, in the mixing scheme, the off-diagonal terms of the $C$ matrix play an important role. In particular, the $\mathrm{CP}$ extended $\mu \tau$ mixing is a novel example of a low energy neutrino mass model, in which even if one does not have any source term for the lepton asymmetry in a particular flavour, a sizeable amount of asymmetry could be injected to that flavour via off-diagonal elements flavour coupling matrix. This in turn emphasizes the importance of the usage of the general structure of flavour coupling matrix (which is assumed to be diagonal in most of the leptogenesis studies) in the Boltzmann equations.

\section{Acknowledgments}

R. Samanta would like to thank Pasquale Di Bari for a useful discussion related to flavour couplings. R. Samanta would like to thank the Royal Society, U.K. and SERB, India for the Newton International Fellowship (NIF).

\section{A General expressions for $\theta_{23}$}

General expression of $\theta_{23}$ for $\mathcal{G}_{1}^{g \mu \tau}$ invariance:

$$
\cos \theta_{23}=\frac{B C \pm \sqrt{B^{2} C^{2}-\left(A^{2}+B^{2}\right)\left(C^{2}-A^{2}\right)}}{A^{2}+B^{2}},
$$

where we define

$$
\begin{aligned}
& A=\sin 2 \theta_{12} \sin \theta_{13} \cos \delta, \\
& B=\sin ^{2} \theta_{12}-\cos ^{2} \theta_{12} \sin ^{2} \theta_{13}, \\
& C=\cos 2 \theta_{g}\left(\sin ^{2} \theta_{13}+\cos ^{2} \theta_{13} \sin ^{2} \theta_{12}\right) .
\end{aligned}
$$

General expression of $\theta_{23}$ for $\mathcal{G}_{2}^{g \mu \tau}$ invariance:

$$
\cos \theta_{23}=\frac{B C \pm \sqrt{B^{2} C^{2}-\left(A^{2}+B^{2}\right)\left(C^{2}-A^{2}\right)}}{A^{2}+B^{2}},
$$

where we define

$$
\begin{aligned}
& A=\sin 2 \theta_{12} \sin \theta_{13} \cos \delta, \\
& B=-\cos ^{2} \theta_{12}+\sin ^{2} \theta_{12} \sin ^{2} \theta_{13}, \\
& C=-\cos 2 \theta_{g}\left(\sin ^{2} \theta_{13}+\cos ^{2} \theta_{13} \cos ^{2} \theta_{12}\right) .
\end{aligned}
$$

Open Access. This article is distributed under the terms of the Creative Commons Attribution License (CC-BY 4.0), which permits any use, distribution and reproduction in any medium, provided the original author(s) and source are credited. 


\section{References}

[1] H. Georgi and S.L. Glashow, Unity of all elementary particle forces, Phys. Rev. Lett. 32 (1974) 438 [INSPIRE].

[2] H. Fritzsch and P. Minkowski, Unified interactions of leptons and hadrons, Annals Phys. 93 (1975) 193 [INSPIRE].

[3] K.S. Babu and R.N. Mohapatra, Predictive neutrino spectrum in minimal SO(10) grand unification, Phys. Rev. Lett. 70 (1993) 2845 [hep-ph/9209215] [INSPIRE].

[4] K.S. Babu and S. Khan, Minimal nonsupersymmetric SO(10) model: gauge coupling unification, proton decay and fermion masses, Phys. Rev. D 92 (2015) 075018 [arXiv:1507.06712] [INSPIRE].

[5] K.S. Babu, B. Bajc and S. Saad, Yukawa sector of minimal SO(10) unification, JHEP 02 (2017) 136 [arXiv:1612.04329] [INSPIRE].

[6] A. Dueck and W. Rodejohann, Fits to SO(10) grand unified models, JHEP 09 (2013) 024 [arXiv:1306.4468] [INSPIRE].

[7] A.S. Joshipura and K.M. Patel, Viability of the exact tri-bimaximal mixing at $M_{\mathrm{GUT}}$ in SO(10), JHEP 09 (2011) 137 [arXiv: 1105.5943] [INSPIRE].

[8] P. Di Bari and L. Marzola, SO(10)-inspired solution to the problem of the initial conditions in leptogenesis, Nucl. Phys. B 877 (2013) 719 [arXiv: 1308.1107] [INSPIRE].

[9] M.K. Parida, B.P. Nayak, R. Satpathy and R.L. Awasthi, Standard coupling unification in $\mathrm{SO}(10)$, hybrid seesaw neutrino mass and leptogenesis, dark matter and proton lifetime predictions, JHEP 04 (2017) 075 [arXiv: 1608.03956] [INSPIRE].

[10] B. Sahoo, M. Chakraborty and M.K. Parida, Neutrino mass, coupling unification, verifiable proton decay, vacuum stability and WIMP dark matter in SU(5), Adv. High Energy Phys. 2018 (2018) 4078657 [arXiv: 1804.01803] [INSPIRE].

[11] M. Fukugita and T. Yanagida, Baryogenesis without grand unification, Phys. Lett. B 174 (1986) 45 [INSPIRE].

[12] A. Riotto and M. Trodden, Recent progress in baryogenesis, Ann. Rev. Nucl. Part. Sci. 49 (1999) 35 [hep-ph/9901362] [INSPIRE].

[13] S. Davidson, E. Nardi and Y. Nir, Leptogenesis, Phys. Rept. 466 (2008) 105 [arXiv:0802.2962] [INSPIRE].

[14] S. Davidson and A. Ibarra, A lower bound on the right-handed neutrino mass from leptogenesis, Phys. Lett. B 535 (2002) 25 [hep-ph/0202239] [INSPIRE].

[15] W. Buchmüller, P. Di Bari and M. Plümacher, Leptogenesis for pedestrians, Annals Phys. 315 (2005) 305 [hep-ph/0401240] [INSPIRE].

[16] K. Moffat, S. Pascoli, S.T. Petcov and J. Turner, Leptogenesis from low energy CP violation, JHEP 03 (2019) 034 [arXiv: 1809.08251] [INSPIRE].

[17] M.J. Dolan, T.P. Dutka and R.R. Volkas, Dirac-phase thermal leptogenesis in the extended type-I seesaw model, JCAP 06 (2018) 012 [arXiv: 1802.08373] [INSPIRE].

[18] T2K collaboration, Updated T2K measurements of muon neutrino and antineutrino disappearance using $1.5 \times 10^{21}$ protons on target, Phys. Rev. D 96 (2017) 011102 [arXiv: 1704.06409] [INSPIRE]. 
[19] T2K collaboration, Combined analysis of neutrino and antineutrino oscillations at T2K, Phys. Rev. Lett. 118 (2017) 151801 [arXiv:1701.00432] [INSPIRE].

[20] NOvA collaboration, Measurement of the neutrino mixing angle $\theta_{23}$ in NOvA, Phys. Rev. Lett. 118 (2017) 151802 [arXiv:1701.05891] [INSPIRE].

[21] NOvA collaboration, Constraints on oscillation parameters from $\nu_{e}$ appearance and $\nu_{\mu}$ disappearance in NOvA, Phys. Rev. Lett. 118 (2017) 231801 [arXiv:1703.03328] [InSPIRE].

[22] A. Himmel, New neutrino oscillation results from NOvA, https://indico.cern.ch/event/696410/, (2018).

[23] MINOS collaboration, Measurement of neutrino and antineutrino oscillations using beam and atmospheric data in MINOS, Phys. Rev. Lett. 110 (2013) 251801 [arXiv:1304.6335] [INSPIRE].

[24] MINOS collaboration, Electron neutrino and antineutrino appearance in the full MINOS data sample, Phys. Rev. Lett. 110 (2013) 171801 [arXiv:1301.4581] [INSPIRE].

[25] RENO collaboration, New results from RENO using 1500 days of data, in $15^{\text {th }}$ International Conference on Topics in Astroparticle and Underground Physics (TAUP 2017), Sudbury, ON, Canada, 24-28 July 2017 [arXiv:1710.08204] [INSPIRE].

[26] I. Esteban, M.C. Gonzalez-Garcia, A. Hernandez-Cabezudo, M. Maltoni and T. Schwetz, Global analysis of three-flavour neutrino oscillations: synergies and tensions in the determination of $\theta_{23}, \delta_{\mathrm{CP}}$ and the mass ordering, JHEP 01 (2019) 106 [arXiv:1811.05487] [INSPIRE].

[27] I. Esteban, M.C. Gonzalez-Garcia, M. Maltoni, I. Martinez-Soler and T. Schwetz, Updated fit to three neutrino mixing: exploring the accelerator-reactor complementarity, JHEP 01 (2017) 087 [arXiv: 1611.01514] [INSPIRE].

[28] P.F. Harrison and W.G. Scott, $\mu-\tau$ reflection symmetry in lepton mixing and neutrino oscillations, Phys. Lett. B 547 (2002) 219 [hep-ph/0210197] [INSPIRE].

[29] W. Grimus and L. Lavoura, A nonstandard CP transformation leading to maximal atmospheric neutrino mixing, Phys. Lett. B 579 (2004) 113 [hep-ph/0305309] [INSPIRE].

[30] R.N. Mohapatra and C.C. Nishi, Implications of $\mu-\tau$ flavored CP symmetry of leptons, JHEP 08 (2015) 092 [arXiv:1506.06788] [INSPIRE].

[31] E. Ma, Neutrino mixing: $A_{4}$ variations, Phys. Lett. B 752 (2016) 198 [arXiv:1510.02501] [INSPIRE].

[32] R. Samanta, P. Roy and A. Ghosal, Consequences of minimal seesaw with complex $\mu-\tau$ antisymmetry of neutrinos, JHEP 06 (2018) 085 [arXiv:1712.06555] [INSPIRE].

[33] G. Altarelli and F. Feruglio, Discrete flavor symmetries and models of neutrino mixing, Rev. Mod. Phys. 82 (2010) 2701 [arXiv: 1002.0211] [INSPIRE].

[34] H. Ishimori, T. Kobayashi, H. Ohki, Y. Shimizu, H. Okada and M. Tanimoto, Non-Abelian discrete symmetries in particle physics, Prog. Theor. Phys. Suppl. 183 (2010) 1 [arXiv: 1003.3552] [INSPIRE].

[35] S.T. Petcov, Discrete flavour symmetries, neutrino mixing and leptonic CP-violation, Eur. Phys. J. C 78 (2018) 709 [arXiv:1711.10806] [InSPIRE]. 
[36] I. Girardi, S.T. Petcov and A.V. Titov, Predictions for the leptonic Dirac CP-violation phase: a systematic phenomenological analysis, Eur. Phys. J. C 75 (2015) 345 [arXiv: 1504.00658] [INSPIRE].

[37] I. Girardi, S.T. Petcov, A.J. Stuart and A.V. Titov, Leptonic Dirac CP-violation predictions from residual discrete symmetries, Nucl. Phys. B 902 (2016) 1 [arXiv: 1509.02502] [INSPIRE].

[38] C.S. Lam, Symmetry of lepton mixing, Phys. Lett. B 656 (2007) 193 [arXiv:0708.3665] [INSPIRE].

[39] C.S. Lam, Determining horizontal symmetry from neutrino mixing, Phys. Rev. Lett. 101 (2008) 121602 [arXiv:0804.2622] [INSPIRE].

[40] S.-F. Ge, D.A. Dicus and W.W. Repko, $Z_{2}$ symmetry prediction for the leptonic Dirac CP phase, Phys. Lett. B 702 (2011) 220 [arXiv:1104.0602] [INSPIRE].

[41] S.-F. Ge, D.A. Dicus and W.W. Repko, Residual symmetries for neutrino mixing with a large $\theta_{13}$ and nearly maximal $\delta_{D}$, Phys. Rev. Lett. 108 (2012) 041801 [arXiv:1108.0964] [INSPIRE].

[42] S.-F. Ge, H.-J. He and F.-R. Yin, Common origin of soft $\mu-\tau$ and CP breaking in neutrino seesaw and the origin of matter, JCAP 05 (2010) 017 [arXiv:1001.0940] [INSPIRE].

[43] H.-J. He and F.-R. Yin, Common origin of $\mu-\tau$ and CP breaking in neutrino seesaw, baryon asymmetry and hidden flavor symmetry, Phys. Rev. D 84 (2011) 033009 [arXiv:1104.2654] [INSPIRE].

[44] Particle Data Group collaboration, Review of particle physics, Chin. Phys. C 38 (2014) 090001 [INSPIRE].

[45] R.N. Mohapatra and S. Nussinov, Bimaximal neutrino mixing and neutrino mass matrix, Phys. Rev. D 60 (1999) 013002 [hep-ph/9809415] [InSPIRE].

[46] T. Fukuyama and H. Nishiura, Mass matrix of Majorana neutrinos, hep-ph/9702253 [INSPIRE].

[47] DAYA BAY collaboration, New measurement of antineutrino oscillation with the full detector configuration at Daya Bay, Phys. Rev. Lett. 115 (2015) 111802 [arXiv: 1505.03456] [INSPIRE].

[48] W. Grimus, A.S. Joshipura, S. Kaneko, L. Lavoura and M. Tanimoto, Lepton mixing angle $\theta_{13}=0$ with a horizontal symmetry $D_{4}, J H E P 07$ (2004) 078 [hep-ph/0407112] [INSPIRE].

[49] W. Grimus, A.S. Joshipura, S. Kaneko, L. Lavoura, H. Sawanaka and M. Tanimoto, Non-vanishing $U_{e 3}$ and $\cos 2 \theta_{23}$ from a broken $Z_{2}$ symmetry, Nucl. Phys. B 713 (2005) 151 [hep-ph/0408123] [INSPIRE].

[50] R.N. Mohapatra and W. Rodejohann, Scaling in the neutrino mass matrix, Phys. Lett. B 644 (2007) 59 [hep-ph/0608111] [INSPIRE].

[51] A.S. Joshipura and W. Rodejohann, Scaling in the neutrino mass matrix, $\mu-\tau$ symmetry and the see-saw mechanism, Phys. Lett. B 678 (2009) 276 [arXiv:0905.2126] [INSPIRE].

[52] R. Samanta, P. Roy and A. Ghosal, Extended scaling and residual flavor symmetry in the neutrino Majorana mass matrix, Eur. Phys. J. C 76 (2016) 662 [arXiv:1604.06731] [INSPIRE]. 
[53] R. Samanta, P. Roy and A. Ghosal, Complex scaling in neutrino mass matrix, Acta Phys. Polon. Supp. 9 (2016) 807 [arXiv: 1604.01206] [InSPIRE].

[54] R. Samanta, M. Chakraborty, P. Roy and A. Ghosal, Baryon asymmetry via leptogenesis in a neutrino mass model with complex scaling, JCAP 03 (2017) 025 [arXiv:1610.10081] [INSPIRE].

[55] R. Sinha, R. Samanta and A. Ghosal, Generalized $Z_{2} \times Z_{2}$ in scaling neutrino Majorana mass matrix and baryogenesis via flavored leptogenesis, JHEP 12 (2017) 030 [arXiv: 1706. 00946] [INSPIRE].

[56] A. Ghosal and R. Samanta, Probing texture zeros with scaling ansatz in inverse seesaw, JHEP 05 (2015) 077 [arXiv: 1501.00916] [INSPIRE].

[57] R. Samanta, M. Chakraborty and A. Ghosal, Evaluation of the Majorana phases of a general Majorana neutrino mass matrix: testability of hierarchical flavour models, Nucl. Phys. B 904 (2016) 86 [arXiv:1502.06508] [InSPIRE].

[58] G.C. Branco, D. Emmanuel-Costa, M.N. Rebelo and P. Roy, Four zero neutrino Yukawa textures in the minimal seesaw framework, Phys. Rev. D 77 (2008) 053011 [arXiv:0712.0774] [INSPIRE].

[59] J. Liao, D. Marfatia and K. Whisnant, Seesaw mechanism with four texture zeros in the neutrino Yukawa matrix, Phys. Rev. D 87 (2013) 073013 [arXiv:1302.2372] [INSPIRE].

[60] G. Ecker, W. Grimus and H. Neufeld, A standard form for generalized CP transformations, J. Phys. A 20 (1987) L807 [InSPIRE].

[61] H. Neufeld, W. Grimus and G. Ecker, Generalized CP invariance, neutral flavor conservation and the structure of the mixing matrix, Int. J. Mod. Phys. A 3 (1988) 603 [INSPIRE].

[62] W. Grimus and M.N. Rebelo, Automorphisms in gauge theories and the definition of CP and P, Phys. Rept. 281 (1997) 239 [hep-ph/9506272] [INSPIRE].

[63] R.N. Mohapatra and C.C. Nishi, $S_{4}$ flavored CP symmetry for neutrinos, Phys. Rev. D 86 (2012) 073007 [arXiv: 1208.2875] [INSPIRE].

[64] S. Gupta, A.S. Joshipura and K.M. Patel, Minimal extension of tri-bimaximal mixing and generalized $Z_{2} \times Z_{2}$ symmetries, Phys. Rev. D 85 (2012) 031903 [arXiv:1112.6113] [INSPIRE].

[65] F. Feruglio, C. Hagedorn and R. Ziegler, Lepton mixing parameters from discrete and $C P$ symmetries, JHEP 07 (2013) 027 [arXiv:1211.5560] [INSPIRE].

[66] M. Holthausen, M. Lindner and M.A. Schmidt, CP and discrete flavour symmetries, JHEP 04 (2013) 122 [arXiv: 1211.6953] [INSPIRE].

[67] M.-C. Chen, M. Fallbacher, K.T. Mahanthappa, M. Ratz and A. Trautner, CP violation from finite groups, Nucl. Phys. B 883 (2014) 267 [arXiv:1402.0507] [InSPIRE].

[68] G.-J. Ding, S.F. King, C. Luhn and A.J. Stuart, Spontaneous CP-violation from vacuum alignment in $S_{4}$ models of leptons, JHEP 05 (2013) 084 [arXiv:1303.6180] [INSPIRE].

[69] G.-J. Ding, S.F. King and A.J. Stuart, Generalised CP and $A_{4}$ family symmetry, JHEP 12 (2013) 006 [arXiv: 1307.4212] [INSPIRE].

[70] F. Feruglio, C. Hagedorn and R. Ziegler, A realistic pattern of lepton mixing and masses from $S_{4}$ and CP, Eur. Phys. J. C 74 (2014) 2753 [arXiv:1303.7178] [InSPIRE]. 
[71] P. Chen, C.-Y. Yao and G.-J. Ding, Neutrino mixing from CP symmetry, Phys. Rev. D 92 (2015) 073002 [arXiv: 1507.03419] [inSPIRE].

[72] C.C. Nishi, New and trivial CP symmetry for extended $A_{4}$ flavor, Phys. Rev. D 93 (2016) 093009 [arXiv: 1601.00977] [INSPIRE].

[73] C.C. Nishi and B.L. Sánchez-Vega, $\mu$ - $\tau$ reflection symmetry with a texture-zero, JHEP 01 (2017) 068 [arXiv: 1611.08282] [INSPIRE].

[74] W. Rodejohann and X.-J. Xu, Trimaximal $\mu-\tau$ reflection symmetry, Phys. Rev. D 96 (2017) 055039 [arXiv : 1705. 02027] [inSPIRE].

[75] R. Samanta and A. Ghosal, Probing maximal zero textures with broken cyclic symmetry in inverse seesaw, Nucl. Phys. B 911 (2016) 846 [arXiv:1507.02582] [INSPIRE].

[76] J.T. Penedo, S.T. Petcov and A.V. Titov, Neutrino mixing and leptonic CP-violation from $S_{4}$ flavour and generalised CP symmetries, JHEP 12 (2017) 022 [arXiv:1705.00309] [INSPIRE].

[77] S.F. King, Unified models of neutrinos, flavour and CP-violation, Prog. Part. Nucl. Phys. 94 (2017) 217 [arXiv: 1701.04413] [INSPIRE].

[78] A. Abada, S. Davidson, A. Ibarra, F.-X. Josse-Michaux, M. Losada and A. Riotto, Flavour matters in leptogenesis, JHEP 09 (2006) 010 [hep-ph/0605281] [INSPIRE].

[79] S. Blanchet and P. Di Bari, Flavor effects on leptogenesis predictions, JCAP 03 (2007) 018 [hep-ph/0607330] [INSPIRE].

[80] P.S.B. Dev, P. Di Bari, B. Garbrecht, S. Lavignac, P. Millington and D. Teresi, Flavor effects in leptogenesis, Int. J. Mod. Phys. A 33 (2018) 1842001 [arXiv:1711.02861] [INSPIRE].

[81] A. Pilaftsis and T.E.J. Underwood, Resonant leptogenesis, Nucl. Phys. B 692 (2004) 303 [hep-ph/0309342] [INSPIRE].

[82] E. Nardi, Y. Nir, E. Roulet and J. Racker, The importance of flavor in leptogenesis, JHEP 01 (2006) 164 [hep-ph/0601084] [INSPIRE].

[83] R. Barbieri, P. Creminelli, A. Strumia and N. Tetradis, Baryogenesis through leptogenesis, Nucl. Phys. B 575 (2000) 61 [hep-ph/9911315] [inSPIRE].

[84] S. Antusch, P. Di Bari, D.A. Jones and S.F. King, A fuller flavour treatment of $\mathrm{N}_{2}$-dominated leptogenesis, Nucl. Phys. B 856 (2012) 180 [arXiv:1003.5132] [INSPIRE].

[85] P. Di Bari and S.F. King, Successful $N_{2}$ leptogenesis with flavour coupling effects in realistic unified models, JCAP 10 (2015) 008 [arXiv:1507.06431] [INSPIRE].

[86] M. Hirsch, S. Morisi, E. Peinado and J.W.F. Valle, Discrete dark matter, Phys. Rev. D 82 (2010) 116003 [arXiv:1007.0871] [InSPIRE].

[87] Y. Hamada, T. Kobayashi, A. Ogasahara, Y. Omura, F. Takayama and D. Yasuhara, Revisiting discrete dark matter model: $\theta_{13} \neq 0$ and $\nu_{R}$ dark matter, JHEP 10 (2014) 183 [arXiv: 1405.3592] [INSPIRE].

[88] W. Grimus and L. Lavoura, Softly broken lepton number $L_{e}-L_{\mu}-L_{\tau}$ with non-maximal solar neutrino mixing, J. Phys. G 31 (2005) 683 [hep-ph/0410279] [INSPIRE].

[89] P.H. Frampton and R.N. Mohapatra, Possible gauge theoretic origin for quark-lepton complementarity, JHEP 01 (2005) 025 [hep-ph/0407139] [INSPIRE]. 
[90] K.S. Babu and R.N. Mohapatra, Predictive schemes for bimaximal neutrino mixings, Phys. Lett. B 532 (2002) 77 [hep-ph/0201176] [INSPIRE].

[91] M.S. Berger and M. Dawid, A Froggatt-Nielsen flavor model for neutrino physics, Int. J. Mod. Phys. A 34 (2019) 1950102 [arXiv:1901.10504] [InSPIRE].

[92] P. Chen, G.-J. Ding, F. Gonzalez-Canales and J.W.F. Valle, Generalized $\mu$ - $\tau$ reflection symmetry and leptonic CP-violation, Phys. Lett. B 753 (2016) 644 [arXiv:1512.01551] [INSPIRE].

[93] R. Sinha, P. Roy and A. Ghosal, CP transformed mixed $\mu-\tau$ antisymmetry for neutrinos and its consequences, Phys. Rev. D 99 (2019) 033009 [arXiv:1809.06615] [INSPIRE].

[94] P. Chen, G.-J. Ding and S.F. King, Leptogenesis and residual CP symmetry, JHEP 03 (2016) 206 [arXiv: 1602.03873] [InSPIRE].

[95] E.W. Kolb and M.S. Turner, The early universe, Front. Phys. 69 (1990) 1 [InSPIRE].

[96] B. Adhikary, M. Chakraborty and A. Ghosal, Flavored leptogenesis with quasidegenerate neutrinos in a broken cyclic symmetric model, Phys. Rev. D 93 (2016) 113001 [arXiv: 1407.6173] [INSPIRE].

[97] PLANCK collaboration, Planck 2018 results. VI. Cosmological parameters, arXiv: 1807.06209 [INSPIRE].

[98] S. Blanchet, P. Di Bari, D.A. Jones and L. Marzola, Leptogenesis with heavy neutrino flavours: from density matrix to Boltzmann equations, JCAP 01 (2013) 041 [arXiv: 1112.4528] [INSPIRE].

[99] K. Moffat, S. Pascoli, S.T. Petcov, H. Schulz and J. Turner, Three-flavored nonresonant leptogenesis at intermediate scales, Phys. Rev. D 98 (2018) 015036 [arXiv:1804.05066] [INSPIRE].

[100] G. Engelhard, Y. Grossman, E. Nardi and Y. Nir, The importance of $N_{2}$ leptogenesis, Phys. Rev. Lett. 99 (2007) 081802 [hep-ph/0612187] [INSPIRE].

[101] D.M. Barreiros, R.G. Felipe and F.R. Joaquim, Combining texture zeros with a remnant $C P$ symmetry in the minimal type-I seesaw, JHEP 01 (2019) 223 [arXiv:1810.05454] [INSPIRE].

[102] R. Samanta and M. Chakraborty, A study on a minimally broken residual TBM-Klein symmetry with its implications on flavoured leptogenesis and ultra high energy neutrino flux ratios, JCAP 02 (2019) 003 [arXiv: 1802.04751] [INSPIRE].

[103] P.S. Bhupal Dev, R. Franceschini and R.N. Mohapatra, Bounds on TeV seesaw models from LHC Higgs data, Phys. Rev. D 86 (2012) 093010 [arXiv:1207.2756] [INSPIRE].

[104] P.S. Bhupal Dev, P. Millington, A. Pilaftsis and D. Teresi, Flavour covariant transport equations: an application to resonant leptogenesis, Nucl. Phys. B 886 (2014) 569 [arXiv: 1404.1003] [INSPIRE].

[105] M. Drewes, B. Garbrecht, D. Gueter and J. Klaric, Testing the low scale seesaw and leptogenesis, JHEP 08 (2017) 018 [arXiv: 1609.09069] [INSPIRE].

[106] B. Garbrecht, Why is there more matter than antimatter? Calculational methods for leptogenesis and electroweak baryogenesis, arXiv:1812.02651 [INSPIRE]. 